\title{
Dimensionierung elektronischer Komponenten in Flurförderzeugen - Untersuchung des Schädigungs- verhaltens unter Laborbedingungen
}

\author{
Dimensioning of Electronic Components in Industrial Trucks - Investigation of \\ the Damage Behavior under Laboratory Conditions
}

\author{
Stefan Weigelt \\ Ludger Overmeyer* \\ Tobias Müller ** \\ Thorsten Schmidt ** \\ * Institut für Transport- und Automatisierungstechnik \\ Leibniz Universität Hannover \\ ** Professur für Technische Logistik \\ Technische Universität Dresden
}

$\mathbf{F}$ lurförderzeuge sind aufgrund ihrer Einsatzbedingungen und konstruktiven Merkmale besonderen Beanspruchungen ausgesetzt. Diese elektrischen, mechanischen und thermischen Beanspruchungen unterscheiden sich teilweise deutlich von denen anderer Fahrzeuge wie Personenwagen oder mobilen Baumaschinen. Um Auslegungs- und Dimensionierungsrichtlinien für die im Flurförderzeug verbauten elektronischen Komponenten zu erarbeiten, wurden an einem Schubmaststapler die auf ausgewählte Komponenten einwirkenden Beanspruchungen aufgezeichnet und umfangreich ausgewertet. In verschiedenen Prüfstandsuntersuchungen wurden die angenommenen Beanspruchungen unter Laborbedingungen nachgestellt, um das Verhalten der Elektronikkomponenten näher zu betrachten und Ausfallcharakteristiken, wie beispielsweise die Zusammenhänge zwischen Belastungshöhe und Belastungshäufigkeit bis zum Ausfall, abzuleiten.

[Schlüsselwörter: Flurförderzeuge, elektronische Komponenten, elektrische und mechanische Belastungen, Schädigung]

$\mathbf{I}$ ndustrial trucks are exposed to special stresses because of their conditions of use and constructive characteristics. These electrical, mechanical and thermal stresses differ significantly from those of other vehicles such as cars or construction machines. In order to develop a design and dimensioning guideline for electronic components which are installed into industrial trucks, the acting stresses were recorded on selected components on a reach truck and evaluated extensively. In various test bench investigations the measured stresses were simulated under laboratory conditions, to get more details about the behavior of electronic components and failure characteristics, such as the relationship between stress level and frequency of stress until failure.

[Keywords: industrial and forklift trucks, electronic components, electrical and mechanical stresses, deterioration]

\section{EINLEITUNG}

Aufgrund ihrer besonders flexiblen Einsatzmöglichkeiten haben Flurförderzeuge für den innerbetrieblichen Materialtransport einen sehr hohen Stellenwert. Aufwendungen für die Wartung und Instandhaltung führen jedoch zu Einbußen der Produktivität ihrer Betreiber. In den vergangenen Jahren sind auf dem Gebiet der Flurförderzeuge dabei vermehrt Ausfälle an elektronischen Komponenten in den Vordergrund getreten, die aufgrund ihrer angestiegenen Komplexität mittlerweile einen erheblichen Einflussfaktor zur Verfügbarkeit des Gesamtsystems Flurförderzeug darstellen [SWSO10]. Um mit Richtlinien und Empfehlungen die Auslegung zu vereinfachen, arbeiten seit Ende 2009 das Institut für Transport- und Automatisierungstechnik (ITA) der Leibniz Universität Hannover zusammen mit dem Institut für Technische Logistik und Arbeitssysteme (TLA) der Technischen Universität Dresden an einer Methodik zur „Dimensionierung von elektronischen Komponenten in Flurförderzeugen“. Dieses über die $\mathrm{AiF}$ geförderte Forschungsvorhaben wird im Rahmen des Programms zur Förderung der Industriellen Gemeinschaftsforschung (IGF) durchgeführt.

Im Fahrbetrieb sind die Elektronikkomponenten von Flurförderzeugen teils deutlich anderen Beanspruchungen als elektronische Komponenten in anderen Einsatzfeldern ausgesetzt. Das ist unter anderem auf die hohe Masse des Fahrzeuges und die geforderte Kippstabilität zurückzufüh- 
ren, wodurch ein nahezu ungedämpftes System resultiert. Hinzu kommen die hohen Anlauf- und Rückspeiseströme, aufgrund häufiger Beschleunigungs- und Bremsvorgänge, die elektrisch betriebene Flurförderzeuge in besonderem Maße beanspruchen. Für die Zuverlässigkeit und Lebensdauer der verwendeten Elektronikkomponenten sind daher vor allem die Einflussgrößen Umgebung und Fahrweg sowie Auslastung und Einsatzintensität von Bedeutung, die auch den definierten Beanspruchungskategorien der VDI-Richtlinie 4.461 [VDI10] zugrunde liegen (siehe Abbildung 1).

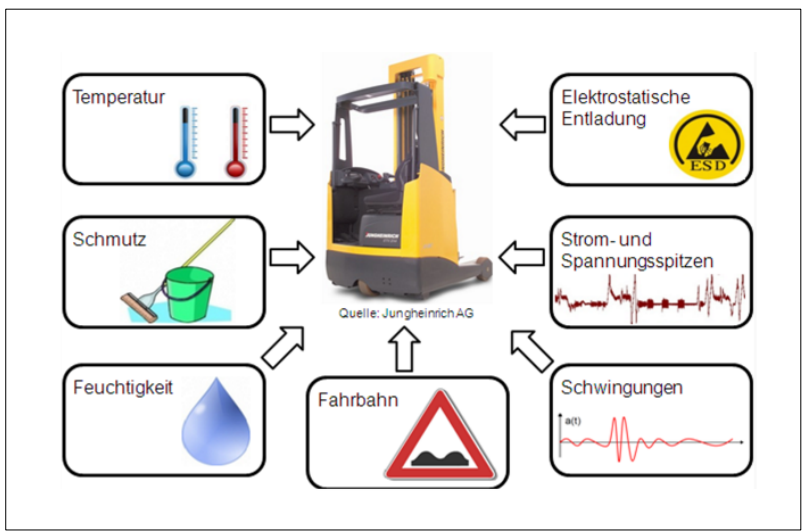

Abbildung 1. Einsatz- und Umgebungsbedingungen von Flurförderzeugen nach VDI 4.461

An einem ausgewählten Schubmaststapler wurden Reihenuntersuchungen an Bauteilen der Leistungselektronik sowie den Bedien- und Anzeigeelementen durchgeführt. Die Auswahl der dabei betrachteten elektronischen Komponenten erfolgte anhand einer Schwachstellenanalyse.

\subsection{SCHWACHSTELLENANALYSE}

Auf Basis von Servicedaten, die von der Jungheinrich AG für unterschiedliche Flurförderzeugtypen zur Verfügung gestellt wurden, sind die Ausfallraten verschiedener in Flurförderzeugen eingesetzter elektronischer und elektro-mechanischer Komponenten statistisch ausgewertet worden. Hierbei wurde nicht nur die Fehlerhäufigkeit einzelner Baugruppen und Komponenten analysiert, sondern auch die Folgen eines Ausfalles betrachtet und entsprechend der zu erwartenden Nutzungsausfallzeit und ihrem Gefährdungspotential gewichtet.

\subsection{AUSWAHL EINES REFERENZ-FLURFÖRDERZEUGES}

In der Schwachstellenanalyse wurde für die weiteren Betrachtungen innerhalb des Forschungsvorhabens ein Schubmaststapler der Jungheinrich AG vom Typ ETV 214 ausgewählt. Abbildung 2 zeigt eine Skizze des maximal $14 \mathrm{~km} / \mathrm{h}$ schnellen und über eine $48 \mathrm{~V}$ Gleichstrombatterie betriebenen Flurförderzeuges.

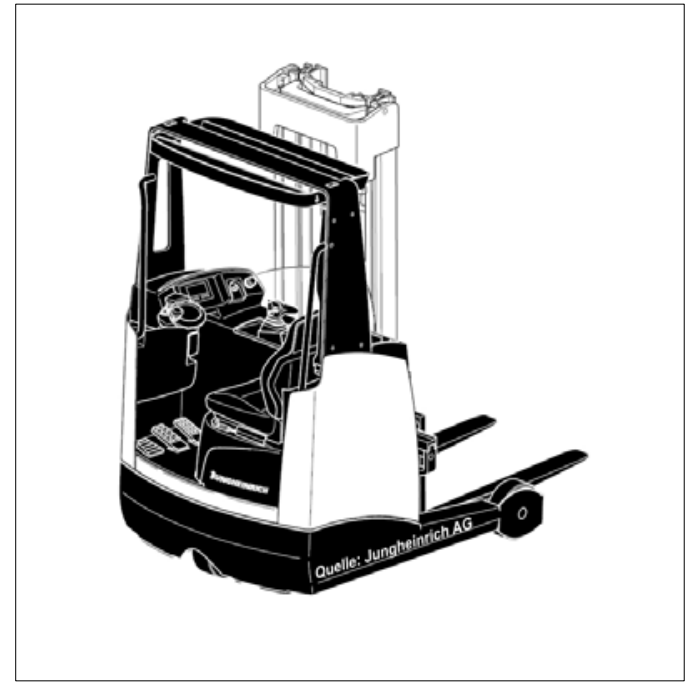

Abbildung 2. Schubmaststapler Jungheinrich ETV 214

$\mathrm{Zu}$ den ausgewählten Komponenten gehören mit der Fahrantriebs- und Hubantriebssteuerung, dem Bordcomputer, dem Bedienhebel des Hubgerüsts und dem Fahrpedal sowohl Komponenten der Leistungselektronik als auch elektronische und mechatronische Bedieneinrichtungen.

Als Komponenten der Leistungselektronik werden die Steuerungsmodule für den Fahr- und Hubantrieb (siehe Abbildung 3) betrachtet, die den Gleichstrom der Traktionsbatterie in Dreiphasen-Wechselstrom für den Fahrund den Hubantrieb umrichten und Sensorsignale in Bezug auf den Betriebszustand des Flurförderzeugs und Bedienerbefehls verarbeiten. Die Fahrantriebssteuerung regelt dazu stufenlos die Drehzahl des Fahrantriebs. Bei einem Ausrollen oder Abbremsen des Fahrzeugs regelt sie zudem die Rekuperation der Bewegungsenergie in die Traktionsbatterie. Die von der Hardware identische Hubantriebssteuerung hält über die Ansteuerung des ebenfalls dreiphasigen Elektromotors zum Antrieb einer Hydraulikpumpe den Betriebsdruck im Hydrauliksystem des Hubmastes auf konstantem Betriebsniveau.

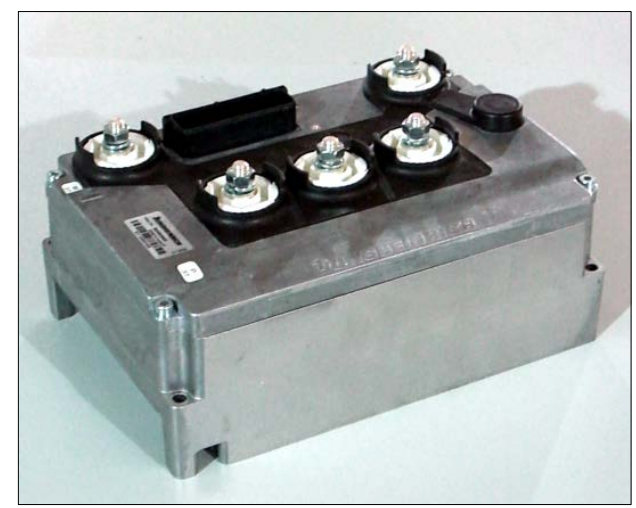

Abbildung 3. Steuerungungsmodul für Fahr- bzw. Hubantrieb 
Mit dem für den Schubmaststapler ETV 214 optionalen Solopilot-Bedienhebel, siehe Abbildung 4, können sämtliche Funktionen des Hubmastes sowie das Signalhorn und der Fahrtrichtungsumschalter betätigt werden. In der Serienausstattung des Staplers wird anstelle des Solopilot-Bedienhebels ein sogenannter Multipilot eingesetzt.

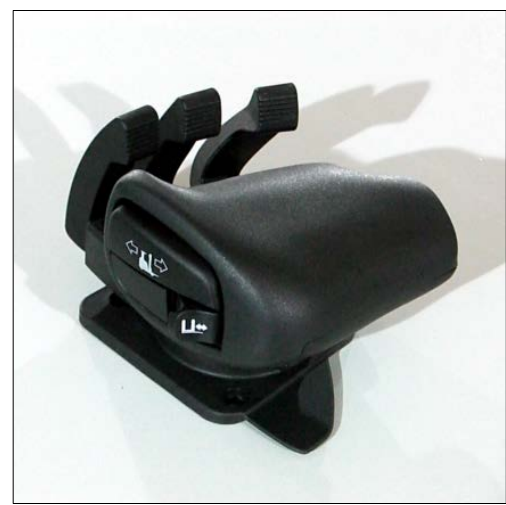

Abbildung 4. Solopilot-Bedienhebel für den Hubmast

Der Bordcomputer (siehe Abbildung 5) zeigt den aktuellen Betriebsstatus des Staplers an - wie beispielsweise auch die Fahrgeschwindigkeit - und ermöglicht die Anwahl diverser Betriebsmodi. Die farbige Darstellung mit Hilfe eines TFT-Bildschirmes ist ebenfalls optional.

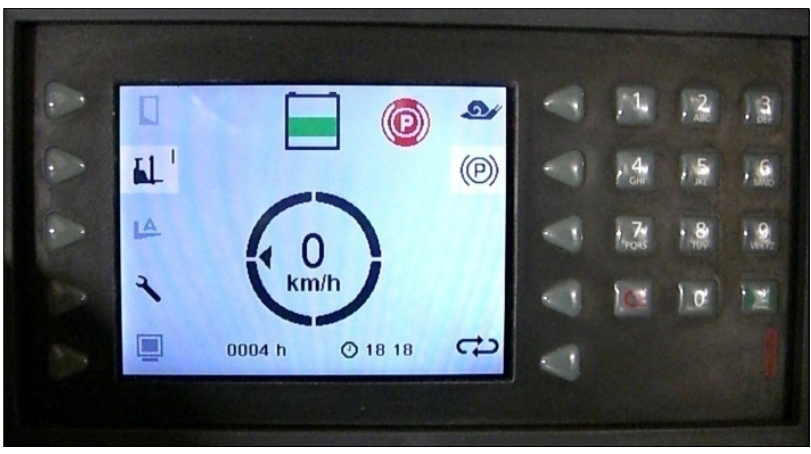

Abbildung 5. Bordcomputer mit Farbdarstellung

Im Fahrpedal (siehe Abbildung 6) wird die aktuelle Stellung mit einem Hall-Sensor erfasst und über die entsprechenden Spannungswerte an die Fahrantriebssteuerung weitergegeben.

\section{AUFNAHME DER BETRIEBSBELASTUNGEN DES FLURFÖRDERZEUGES}

Für die Aufnahme von elektrischen Kenngrößen, wie Ströme und Spannungen an den Komponenten der Leistungselektronik und den Bedieneinrichtungen, wurde ein modulares Messdatenerfassungssystem verwendet. Ein Triaxial-Beschleunigungssensor erfasst zudem die Beschleunigungen in allen drei Raumrichtungen und wurde während der umfangreichen Versuchsreihen an den verschiedenen betrachteten Komponenten angebracht (siehe Abbildung 7).

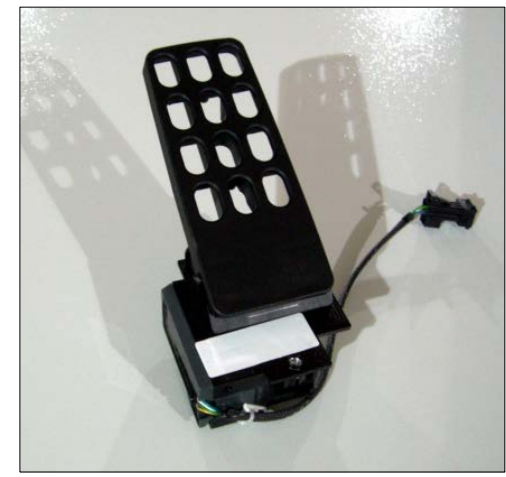

Abbildung 6. Fahrpedal des Schubmaststaplers

Durch die Verwendung eines Dualkamerasystems wurde während der Versuchsmessungen der Fahrweg und Bedienerplatz in Form von Videoaufzeichnungen dokumentiert. Damit war es nach den Versuchsreihen möglich, charakteristische Verläufe in den Messschrieben den jeweiligen Fahrzuständen und Bedienmanövern zuzuordnen.

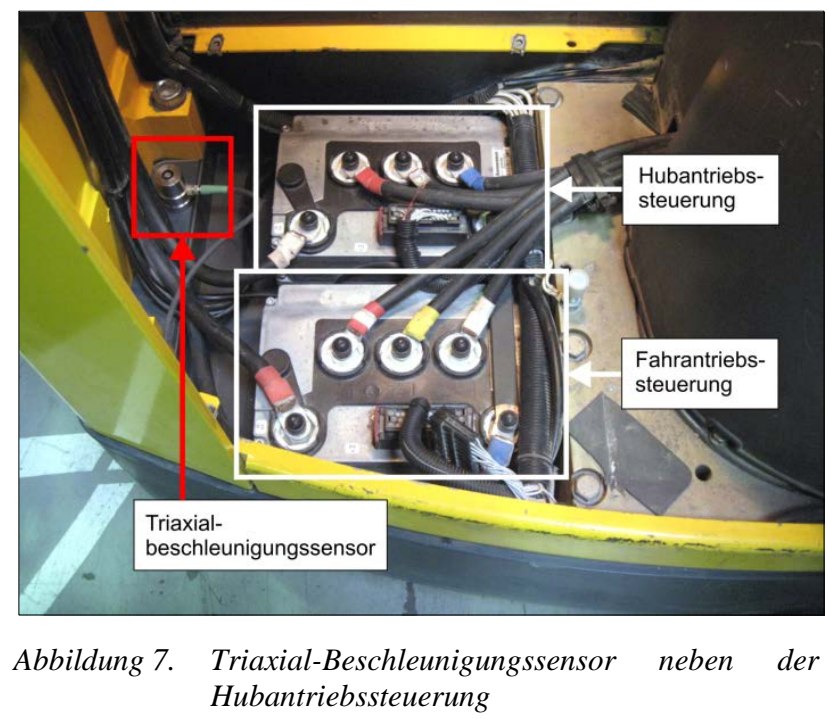

\subsection{ARBEITSSPIELE UND TESTZYKLEN}

Zur Aufnahme der im Flurförderzeugbetrieb auf die ausgewählten Komponenten einwirkenden Belastungen unter reproduzierbaren Bedingungen wurden genormte und aus der Literatur bekannte sowie selbst erarbeitete Arbeitsspiele verwendet.

Das Arbeitsspiel der VDI-Richtlinie 2.198 ist in Abbildung 8 dargestellt. Es besteht aus der Fahrt zwischen den beiden Punkten A und B, an denen die bei der Versuchsdurchführung aufgenommene Nutzmasse um jeweils zwei Meter angehoben und wieder abgesenkt wird. Die VDI-Richtlinie gibt dabei vor, dass die Versuchsfahrten mit maximaler Nutzmasse erfolgen müssen. Dieses entspricht beim verwendeten Schubmaststapler $1.400 \mathrm{~kg}$. Als Abwandlung von dieser Richtlinie wurden zusätzlich auch Versuchsmessungen ohne Nutzmasse oder mit variierter Nutzmasse durchgeführt. 


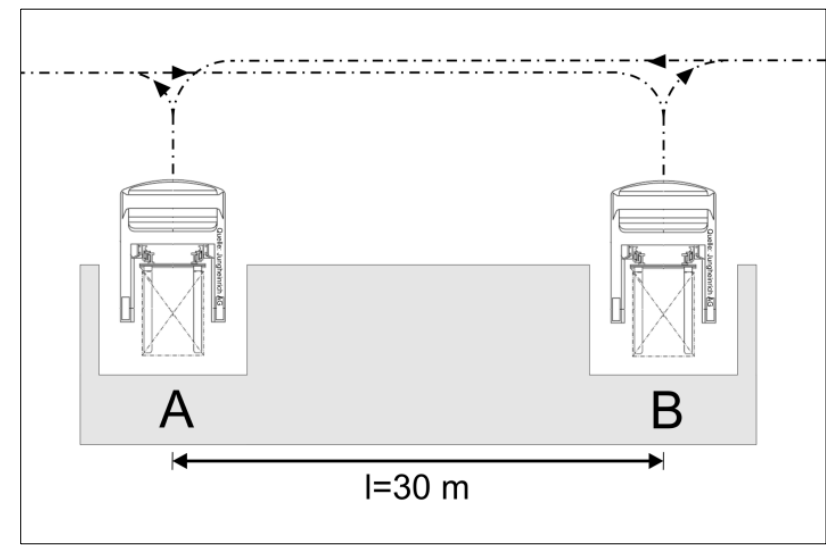

Abbildung 8. Arbeitsspiel nach VDI-Richtlinie 2.198

Das in Abbildung 9 gezeigte Egberts-Fahrprofil wurde von Theo Egberts im Rahmen seiner Tätigkeit bei der Zeitschrift „Transport + Opslag“ aufgestellt und wird ebenfalls vom Magazin „Fördern und Heben“ zum Test von Flurförderzeugen angewendet [Egb00]. Dieser Testzyklus beinhaltet als Abwandlung des Arbeitsspieles der VDI-Richtline 2198 eine Schleuse, die auf dem zurückzulegenden Weg zwischen den Punkten A und B stets durchfahren wird. Die Fahrmanöver beinhalten dabei sowohl mehrfache Vorwärtsfahrten als auch eine Rückwärtsfahrt, die sowohl mit als auch ohne Nutzmasse absolviert werden. Die zu transportierenden Massen betragen dabei ein Drittel und zwei Drittel der maximalen Nutzmasse. Auch bei diesen Untersuchungen wurden als Variation weitere verschiedene Nutzmassen verwendet.

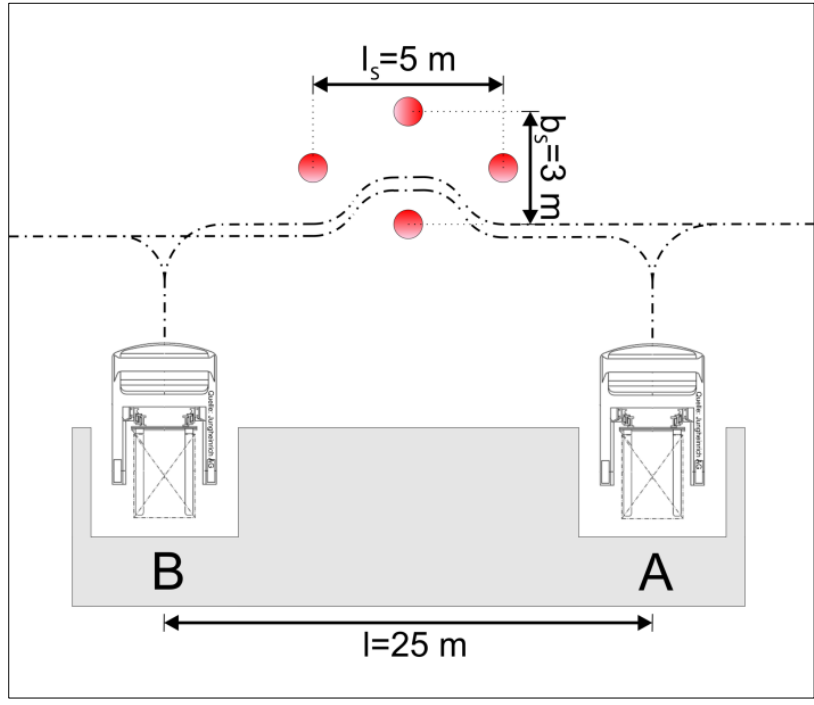

Abbildung 9. Testzyklus nach Theo Egberts

Zusätzlich zu diesen bekannten Fahrzyklen wurden Kundennutzungsdaten zum Schubmaststapler der Baureihe 2 statistisch ausgewertet. Aus diesen durch die Jungheinrich AG zur Verfügung gestellten Daten konnten statistisch repräsentative Fahr- und Lastszenarien aufgestellt werden. Das ITA-Fahrprofil (ITA-F) besteht wie in Abbildung 10 illustriert aus verschiedenen Streckenabschnit- ten, die mit unterschiedlicher Geschwindigkeit befahren werden.

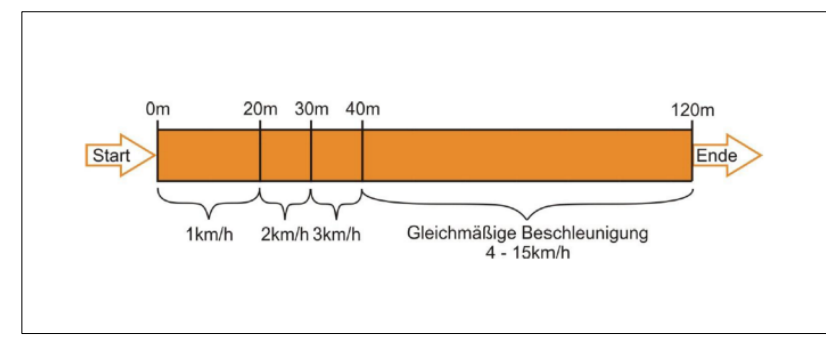

Abbildung 10. Fahrprofil des ITA (ITA-F)

Ergänzend zu dem Fahrszenario wurde ein Rangierszenario (ITA-R) entwickelt, wie es in Abbildung 11 dargestellt ist.

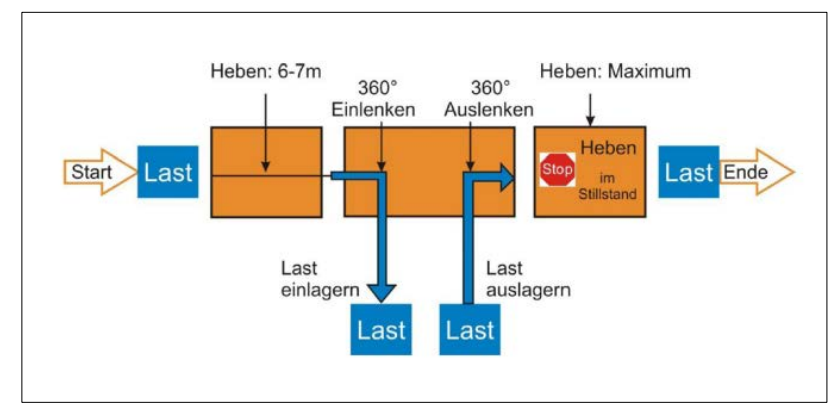

Abbildung 11. Rangierszenario des ITA (ITA-R)

Hierbei schlägt der Bediener bei langsamer Fahrgeschwindigkeit das Lenkrad um $360^{\circ}$ ein und dreht den Stapler um $90^{\circ}$. Anschließend wird ein Einlagerungsprozess simuliert, indem die Nutzmasse auf 1,8 m angehoben und wieder abgesenkt wird. Danach wird der Stapler mit einer Drehung um $90^{\circ}$ zurückgesetzt, bevor die Nutzmasse im Stillstand auf Maximalhöhe angehoben und schließlich abgesetzt wird. Das gesamte ITA-Testprofil ergibt sich aus der Kombination der beschriebenen Fahr- und Rangierszenarien. Diese werden je nach erwarteter Kundennutzung zu unterschiedlichen Teilen zusammengesetzt.

Zusätzlich zu den genannten Fahrprofilen wurde durch Nachstellung von Schwellenüberfahrten eine in der Praxis häufig auftretende besondere Beanspruchungssituation nachgestellt. Dabei wurde sich bei den Untersuchungen zunächst an dem Versuchsaufbau der DIN EN 13.059 „Sicherheit von Flurförderzeugen - Schwingungsmessung“ orientiert, die sich mit der Schwing- und Stoßbelastung auf den Flurförderzeugbediener (Humanschwingungen) auseinandersetzt [DIN09]. Dieser Aufbau sieht in der entsprechenden Testklasse für Schubmaststapler mit einem mittleren Raddurchmesser von mehr als 200 mm eine zu überfahrende Schwellenhöhe $h$ von $5 \mathrm{~mm}$ und eine Fahrgeschwindigkeit $v$ von $5 \mathrm{~km} / \mathrm{h}$ vor. Im weiteren Verlauf der Versuchsmessungen wurde die Schwellenhöhe von $3 \mathrm{~mm}$ bis $11 \mathrm{~mm}$ und die Fahrgeschwindigkeit von $3 \mathrm{~km} / \mathrm{h}$ bis $14 \mathrm{~km} / \mathrm{h}$ stufenweise variiert. 
Einen Überblick über die ermittelten Kenngrößenverläufe und die während der Versuchsreihen aufgetretenen Maximalbeanspruchungen gibt der nachfolgende Abschnitt.

\subsection{Auswertung DER MESSDAten}

Für die Auswertung der Messdaten sei vorangestellt, dass für die anschließende Nachbildung der Beanspruchungen am Schwingprüfsystem (vgl. Abschnitt 3.2) insbesondere die vertikalen Beschleunigungen von Interesse sind. Daher wird in dieser Auswertung auch nur auf diese Beschleunigungen eingegangen. Während der Reihenuntersuchungen wurde in den meisten Fällen ein TriaxialBeschleunigungssensor auf dem Rahmen neben der Fahrantriebssteuerung als Referenzgröße platziert (vgl. Abbildung 7, S. 3). Der zweite Triaxial-Beschleunigungssensor wurde an einer weiteren zu prüfenden Komponente befestigt, z. B. dem Fahrpedal oder dem Bedienhebel.

Die in der Auswertung angegebenen maximalen Beschleunigungen ergeben sich aus dem arithmetischen Mittel mehrerer Reihenmessungen, von denen jeweils der maximale Betrag erfasst wurde. Einen Überblick über die nach diesem Verfahren ermittelten Maximalbeschleunigungen zeigt Abbildung 12.

Dabei bedeuten:

- SW: Schwellenfahrt

- FP: Fahrpedal

- BC: Bordcomputer

- $\quad$ BH: Bedienhebel

- FS: Fahrsteuerung

Für die Schwellenüberfahrten ist hinzuzufügen, dass für die Benennung der Testbedingungen, jeweils auf der Abszisse aufgetragen, folgende Reihenfolge gilt: Nutzmasse, Schwellenhöhe, Geschwindigkeit. Der Index für das Fahrpedal (links) beschreibt in Abbildung 12: Schwellenfahrt mit einer Nutzmasse von $600 \mathrm{~kg}$, einer Schwellenhöhe von $11 \mathrm{~mm}$ unter maximal möglicher Geschwindigkeit.

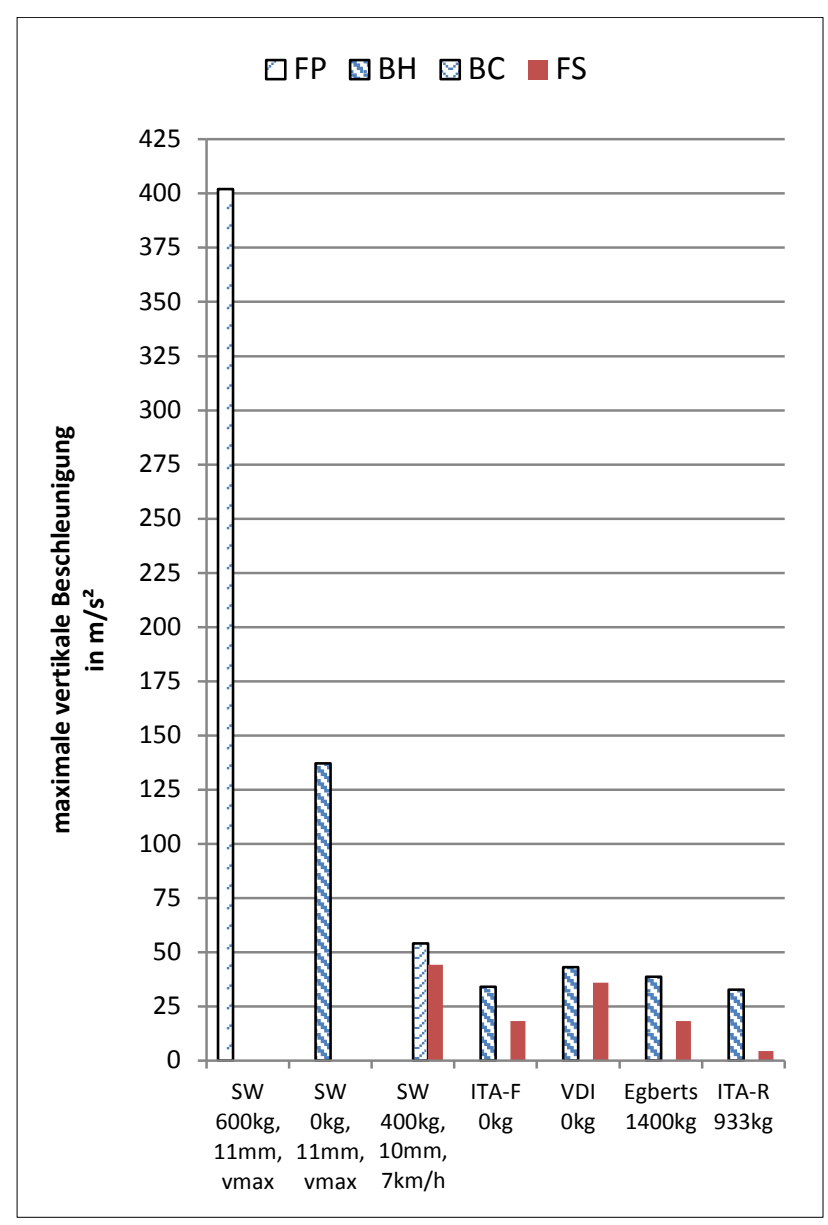

Abbildung 12. Gemessene Maximalbeschleunigungen innerhalb der geprüften Testszenarien

Für die Untersuchungen in diesem Projekt sind nicht nur die Beträge der gemessenen Beschleunigungen von großem Interesse, sondern vor allem der Ort für die maximal auftretende Beschleunigung. Dieser ist in den nachfolgenden Abbildungen in den jeweiligen Beschleunigungsverläufen markiert. Im VDI-Arbeitsspiel tritt die maximale vertikale Beschleunigung der Fahrantriebssteuerung beim Ausfahren des Hubgerüsts auf, beim Bedienhebel vom Übergang aus der maximalen Geschwindigkeit des Staplers in den Verzögerungszustand, wie aus Abbildung $13 \mathrm{zu}$ entnehmen ist. In dieser Abbildung sind im Kenngrößenverlauf des Stromes der Hubantriebssteuerung die einzelnen nacheinander ausgeführten Arbeitsschritte zu erkennen, die folgende Arbeitsbewegung widerspiegeln: Hubmast senkrecht stellen, Schubmast ausfahren, Nutzmasse anheben, Nutzmasse senken, Schubmast einfahren und Hubgerüst in Fahrposition neigen. Diese Arbeitsabfolge ist in den weiterführenden Abbildungen, außer bei Abbildung 15, ebenfalls zu erkennen. 


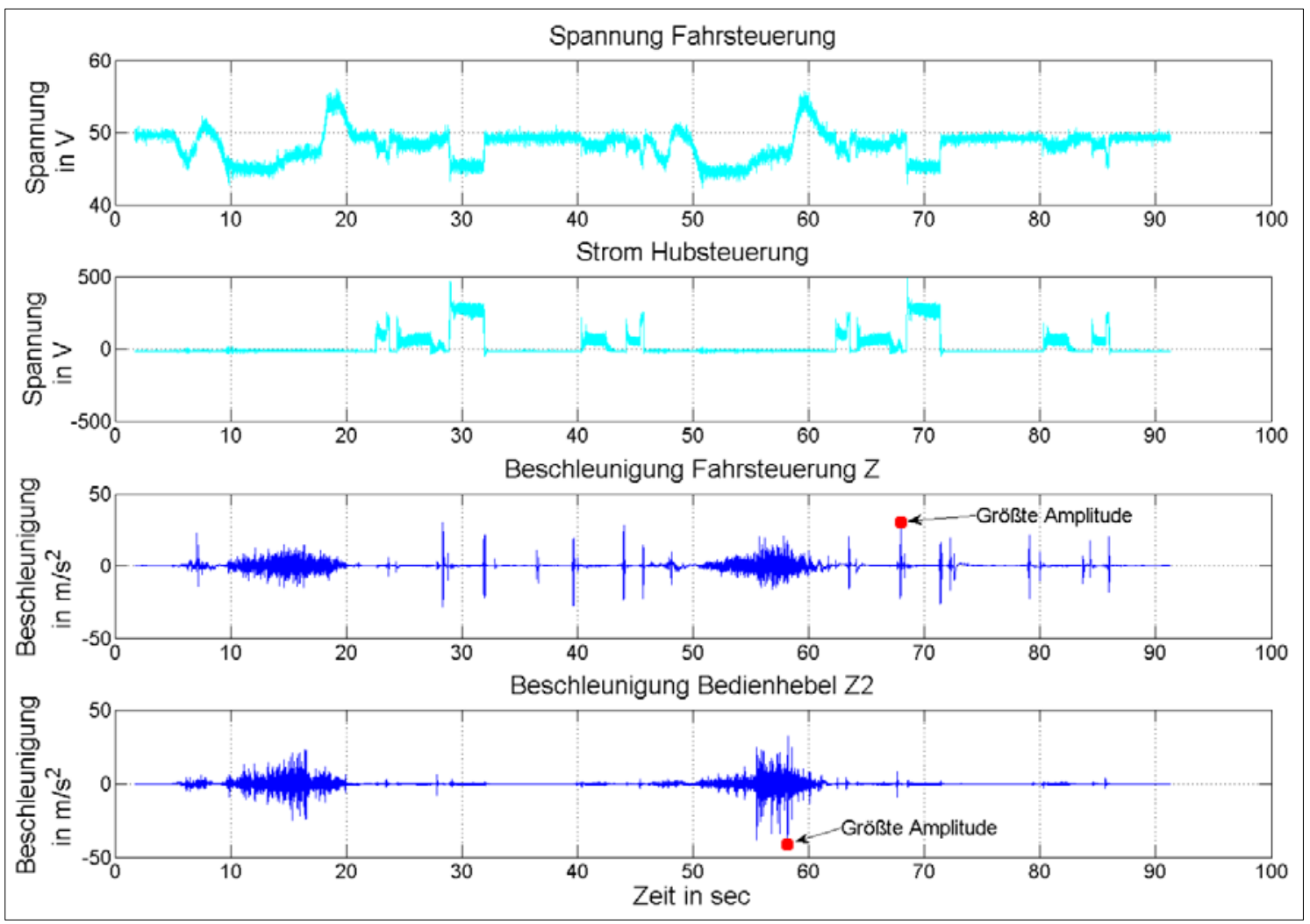

Abbildung 13. Kenngrößenverläufe bei einem VDI-Arbeitsspiel mit 0 kg Nutzmasse

Beim Testzyklus nach Egberts sind die höchsten Beschleunigungswerte der Fahrantriebssteuerung während der Schleusendurchfahrt entstanden, die ohne Nutzmasse absolviert wurde. Beim Bedienhebel des Hubgerüstes sind die höchsten Beschleunigungen beim Neigen des Hubgerüstes aufgetreten (siehe Abbildung 14).

Während des ITA-Fahrprofils (ITA-F) treten die maximalen Beschleunigungen an der Fahrantriebssteuerung sowie dem Bedienhebel des Hubgerüstes beim Beginn der Verzögerung aus maximaler Fahrgeschwindigkeit auf (siehe Abbildung 15).
Beim ITA-Rangierprofil (ITA-R), bei dem die Eigenschaft des Rangierens im Vordergrund steht, sind trotz der geringen Fahrgeschwindigkeit von $3 \mathrm{~km} / \mathrm{h}$ die maximalen Beschleunigungen an der Fahrantriebssteuerung beim langsamen Vorwärtsfahren während des Einlenkens aufgetreten. Beim Bedienhebel des Hubgerüstes entstanden die höchsten Beschleunigungswerte wieder beim Ankippen des Hubgerüstes in die leicht geneigte Fahrposition (siehe Abbildung 16). 


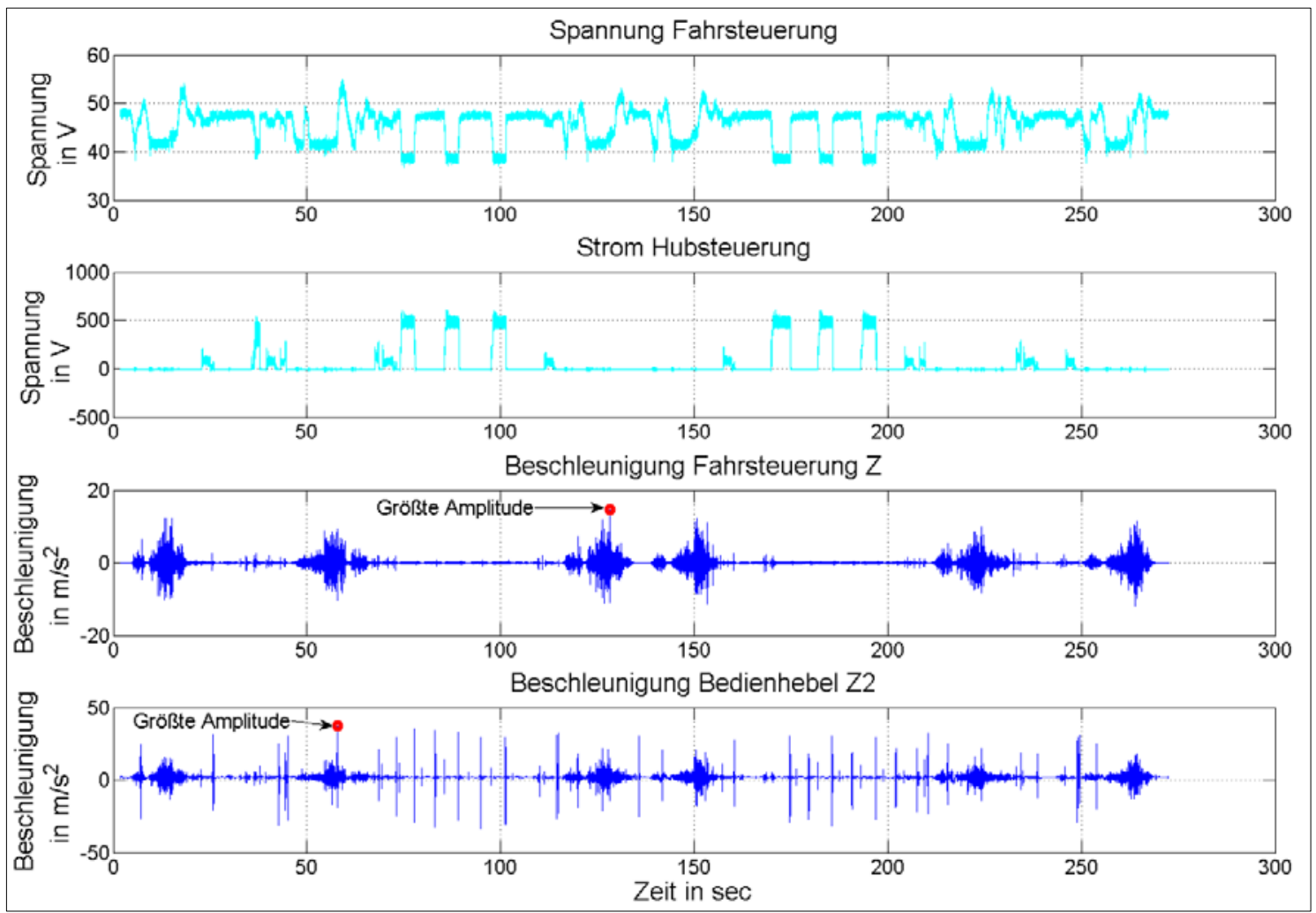

Abbildung 14. Egberts-Testzyklus mit 1.400 kg Nutzmasse
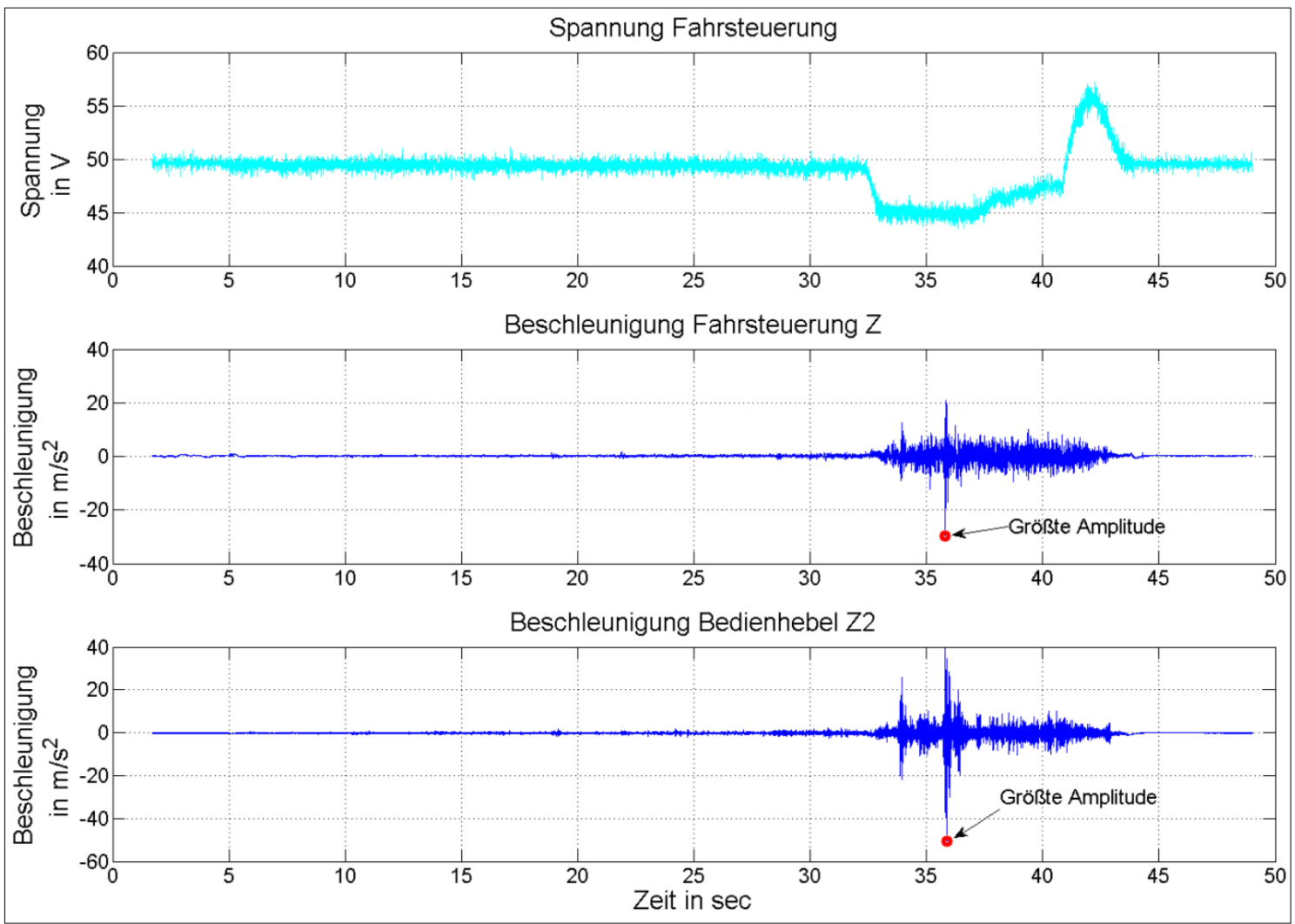

Abbildung 15. Kenngrößenverläufe bei einem ITA Fahrprofil mit 0 kg Nutzmasse 


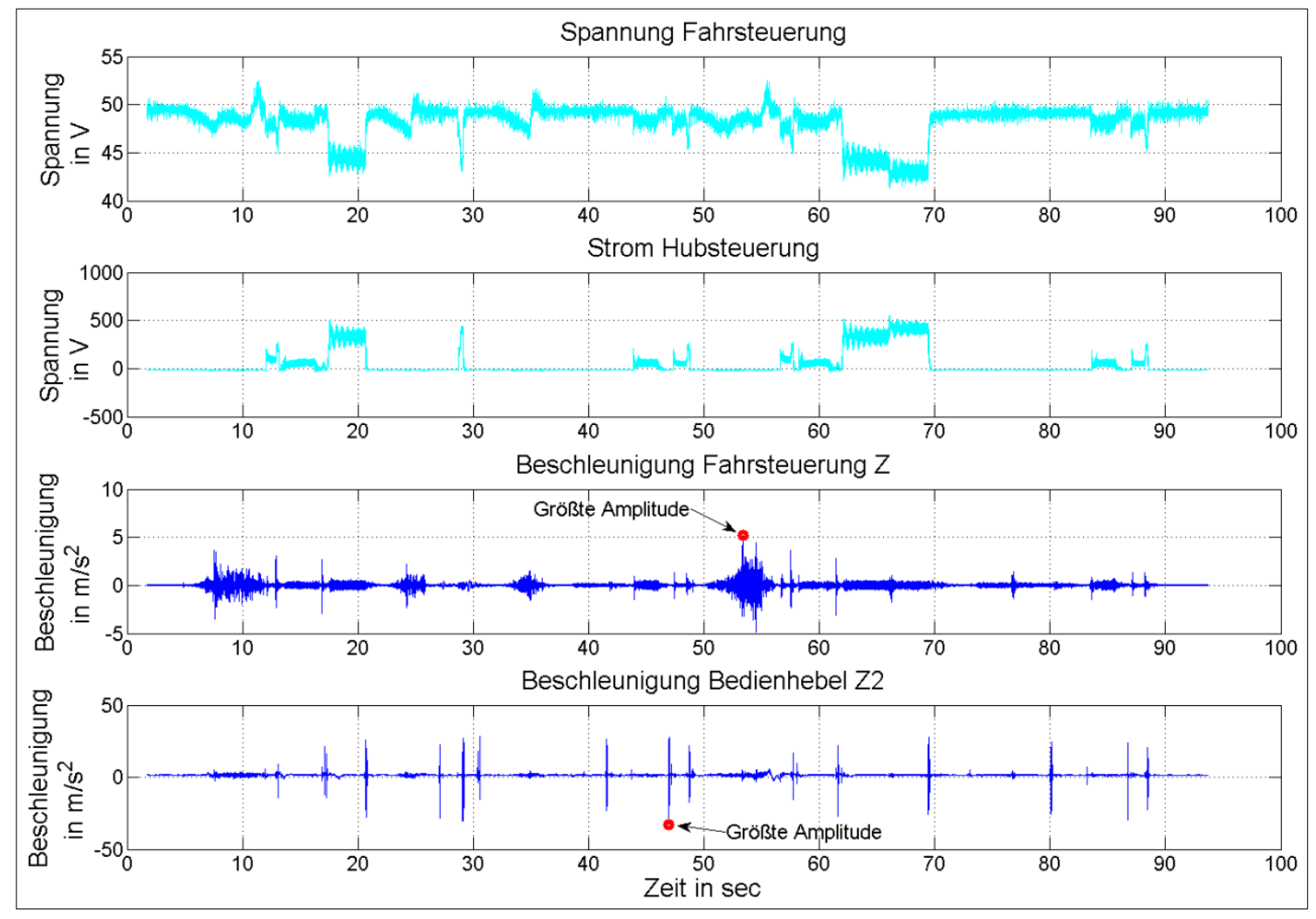

Abbildung 16. ITA Rangierprofil (ITA-R) mit $933 \mathrm{~kg}$ Nutzmasse

Interessanterweise sind die maximalen Beschleunigungen nie beim Heben in die maximale Hubposition oder beim Senken aus dieser Position heraus zu finden, obwohl der Teleskop-Hubmast bei diesen Vorgängen einen für den Bediener deutlich spürbaren Stoß verursacht. Wie aus Abbildung $12 \mathrm{zu}$ entnehmen ist, verursachen die Überfahrten von $11 \mathrm{~mm}$ hohen Fahrbahnschwellen mit maximaler Fahrgeschwindigkeit am Bedienhebel mehr als die 3-fache Beschleunigung gegenüber dem Testszenario der VDI-Richtlinie 2.198. Bereits ab einer Schwellenhöhe von $5 \mathrm{~mm}$ und maximaler Fahrgeschwindigkeit werden bei Schwellenüberfahrten die maximalen Beschleunigungswerte von VDI und Egberts am Bedienhebel erreicht. Daher sollen diese Schwellenüberfahrten im weiteren Verlauf der Untersuchungen genauer betrachtet werden. Abbildung 17 zeigt anhand eines Beispiels die auftretende Beschleunigung orthogonal zur Fahrbahn und die umgesetzte Leistung an der Fahrmotorsteuerung für die Überfahrt einer $7 \mathrm{~mm}$ hohen Schwelle mit einer Geschwindigkeit von $7 \mathrm{~km} / \mathrm{h}$. Am Verlauf der gemessenen Beschleunigung ist das Auftreffen des Antriebsrades auf die Fahrbahnschwelle zum Zeitpunkt $\mathrm{t}=15,4 \mathrm{~s}$ und der Lasträder bei $\mathrm{t}=16,2 \mathrm{~s}$ deutlich $\mathrm{zu}$ erkennen. Anhand des aufgezeichneten Verlaufes der umgesetzten Leistung an der Fahrmotorsteuerung wird das Nachregeln der Fahrantriebssteuerung zum Überwinden des Hindernisses zu diesem Zeitpunkt ersichtlich. Somit lässt sich auch die Korrelation der verschiedenen Beanspruchungsarten gut erkennen. Die aufgezeichneten Beanspruchungen wurden anschließend sowohl im Zeit- als auch im Frequenzbe- reich analysiert und ausgewertet. Abbildung 18 zeigt einen Vergleich der maximal auftretenden vertikalen Beschleunigungen für unterschiedliche Fahrgeschwindigkeiten und Schwellenhöhen bei der maximalen Nutzmasse von $1.400 \mathrm{~kg}$. Aus dem Diagramm wird ersichtlich, dass neben der Geschwindigkeit auch die Höhe der Fahrbahnschwellen die vertikalen Maximalbeschleunigungen erheblich beeinflusst. Die jeweils größten Beschleunigungen an den betrachteten Komponenten wurden entsprechend bei maximaler Geschwindigkeit $(14 \mathrm{~km} / \mathrm{h})$ und größter Schwellenhöhe (11 mm) ermittelt. An den Steuerungsmodulen für Fahr- und Hubantrieb traten die höchsten Beschleunigungswerte von $49 \mathrm{~m} / \mathrm{s}^{2}$ ohne aufgenommene Nutzmasse auf. 


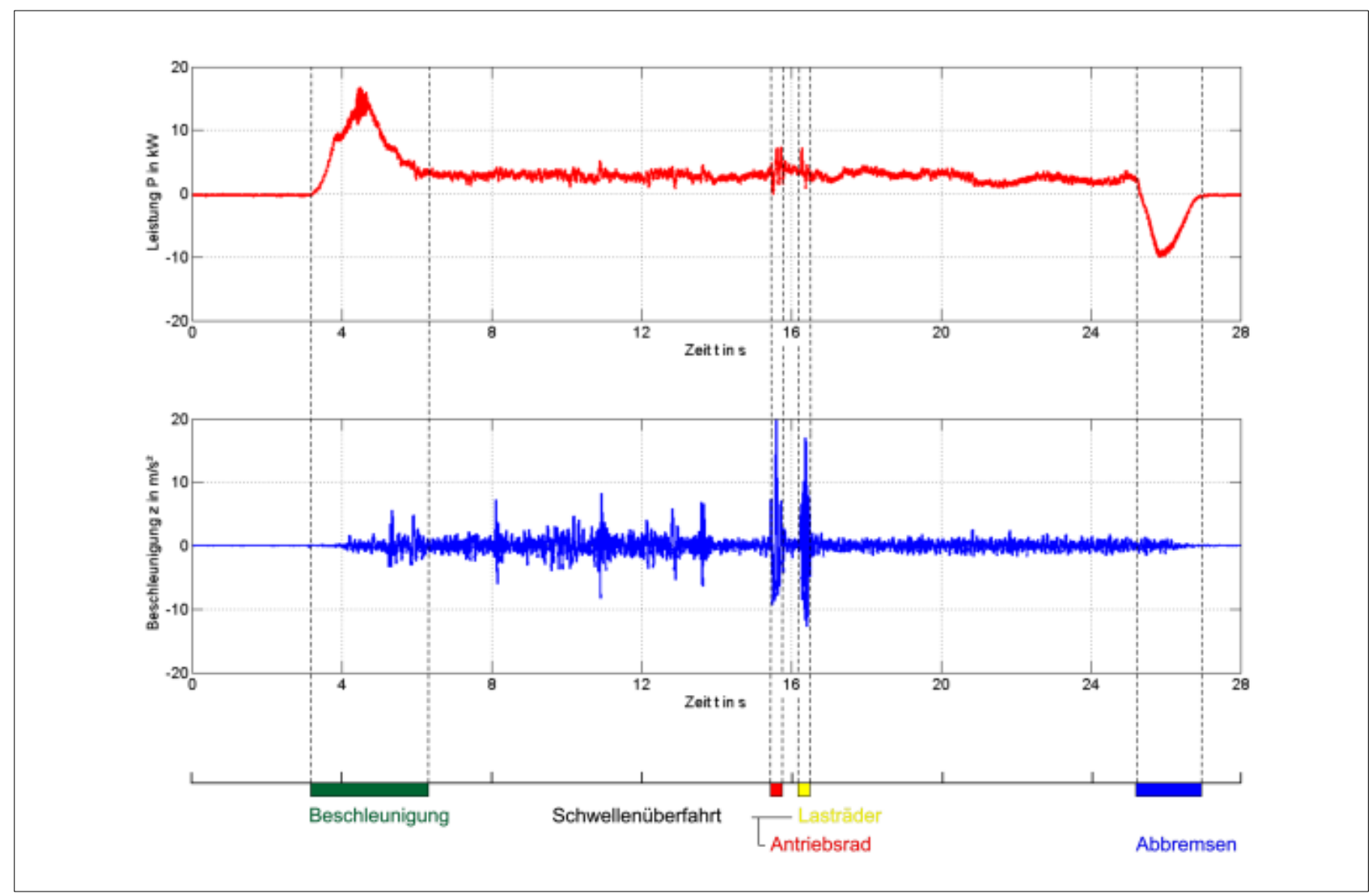

Abbildung 17. Beispiel einer Schwellenfahrt mit: 1.400 kg, 7 mm Schwellenhöhe, 7 km/h

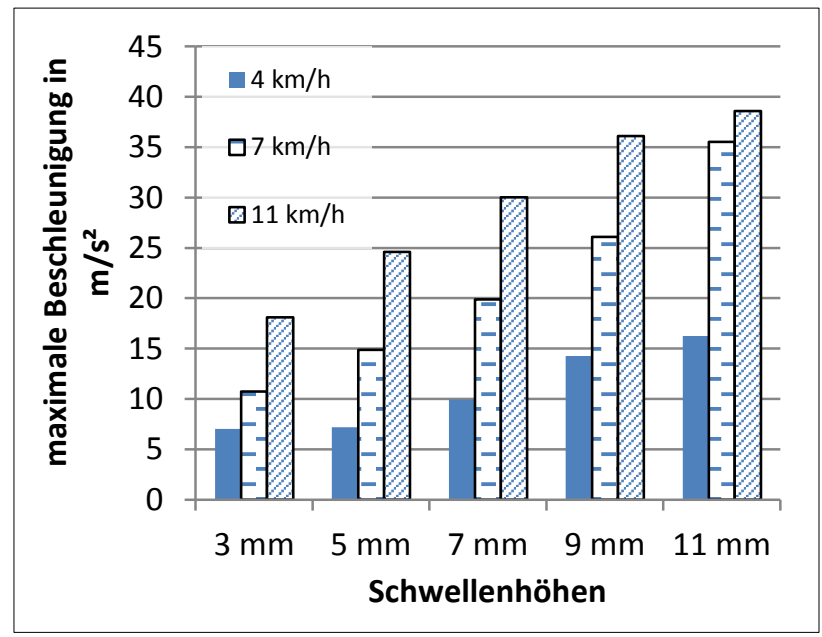

Abbildung 18. Maximalbeschleunigungen der Fahrantriebssteuerung mit variierten Geschwindigkeiten sowie Schwellenhöhen und einer Nutzmasse von $1.400 \mathrm{~kg}$

Für den Bedienhebel des Hubgerüsts wurden bei Versuchen mit der größtmöglichen zulässigen Nutzmasse von $1.400 \mathrm{~kg}$ sogar Maximalbeschleunigungen von bis zu $136 \mathrm{~m} / \mathrm{s}^{2}$ aufgenommen. Die Variation der Nutzmasse führte in Bezug auf den Bedienhebel des Hubgerüsts zu einer Veränderung des Schwingungssystems. Die jeweils resultierenden vertikalen Maximalbeschleunigungen auf diese Komponente sind in Abbildung 19 für unterschied- liche Nutzmassen und die Fahrt mit $7 \mathrm{~km} / \mathrm{h}$ über eine $5 \mathrm{~mm}$ hohe Bodenschwelle dargestellt.

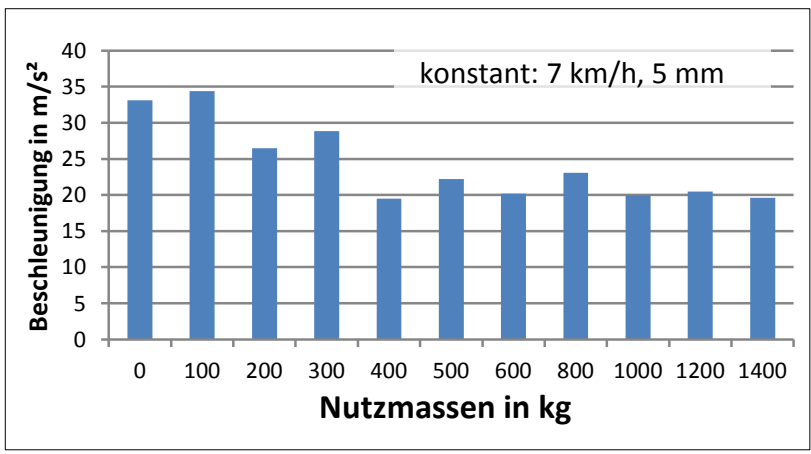

Abbildung 19. Veränderung des Schwingungsverhaltens bei gleichbleibender Fahrgeschwindigkeit und Schwellenhöhe unter Variation der Nutzmasse

Das Fahrpedal wurde während der Versuche von allen betrachteten Komponenten mit den höchsten Schwingund Stoßbelastungen beansprucht. Die maximale Beschleunigung von $400 \mathrm{~m} / \mathrm{s}^{2}$ trat bei den Versuchsfahrten mit einer Nutzmasse von $600 \mathrm{~kg}$, maximal möglicher Geschwindigkeit und einer Schwellenhöhe von $11 \mathrm{~mm}$ auf.

Da der Bordcomputer nur bei Messungen mit 7 km/h untersucht wurde, wird nachfolgend lediglich ein Überblick über die drei elektro-mechanischen Bedieneinrichtungen gegeben. So sind bei definierter Geschwindigkeit 
von $7 \mathrm{~km} / \mathrm{h}$ und einer vorgegebenen Schwellenhöhe von $10 \mathrm{~mm}$ folgende maximale Beschleunigungen in einem Vergleichstest gemessen worden:

- $\quad$ Bordcomputer $-54 \mathrm{~m} / \mathrm{s}^{2}$

- $\quad$ Bedienhebel - $66 \mathrm{~m} / \mathrm{s}^{2}$

- $\quad$ Fahrpedal - $126 \mathrm{~m} / \mathrm{s}^{2}$.

\section{BEWERTUNG UND NACHSTELLUNG DER MECHANISCHEN BELASTUNGEN}

Nach den ermittelten Belastungen für die jeweilige Komponente wurde untersucht, ob die Testszenarien mit den höchsten Beschleunigungen auch die größten Schädigungen hervorrufen. Hierzu wird eine Gesamtbetrachtung der Messfahrten vorgenommen. Dadurch werden auch die Bewegungsvorgänge mit weniger ausgeprägten Beschleunigungsspitzen - wie z. B. Verzögern, Beschleunigen, Hub- und Senkvorgänge sowie das Kippen des Hubmasts - einer Fahrt erfasst, die ebenfalls zur Schädigung beitragen können.

\subsection{BEWERTUNG DES SCHÄDIGUNGSVERHALTENS}

Um auf Basis der ermittelten mechanischen Beanspruchungsverläufe die erwartete Lebensdauer der betrachteten Komponenten quantitativ miteinander vergleichen zu können, wurde die aus der Betriebsfestigkeit bekannte Rainflow-Klassierung herangezogen. Der Algorithmus dieses zweiparametrischen Klassierverfahrens orientiert sich an der Vorstellung, dass ein Regenfluss über die in senkrechter Richtung dargestellte Beschleunigungs-Zeit-Funktion abläuft. Für diese Regenflüsse gibt es definierte Mischungs- und Stopp-Regeln, die eine Erkennung der Schwingspiele ermöglichen. Dabei werden Hysteresen geschlossen, die als Schädigungsereignis zu bewerten sind. Aus der daraus entstehenden RainflowMatrix wird für jedes Schwingspiel ein Ersatzschwingspiel für die Bewertung der Schädigung gebildet. Dieses besteht aus der Summe aller Schwingspiele und mittleren Schwingspielen der jeweiligen Klasse:

$\mathrm{S}_{\mathrm{a}_{\mathrm{ers}, \mathrm{i}, \mathrm{j}}}=\sum_{\mathrm{i}}\left(\mathrm{S}_{\mathrm{ai}, \mathrm{j}}+\mathrm{M} \cdot \mathrm{S}_{\mathrm{m}_{\mathrm{i}, \mathrm{j}}}\right)$

$\begin{array}{cl}\text { mit } \mathrm{S}_{\mathrm{a}, \mathrm{ers}, \mathrm{i}, \mathrm{j}} & \text { Ersatzschwingspiel } \\ \mathrm{S}_{\mathrm{a}, \mathrm{i}, \mathrm{j}} & \text { Schwingspiel } \\ \mathrm{M} & \text { Mittelspannungseinflussfaktor } \\ \mathrm{S}_{\mathrm{m}, \mathrm{i}, \mathrm{j}} & \text { Mittelwert des Schwingspiels } \\ \mathrm{i}, \mathrm{j} & \text { Klassengrenze des Schwingspiels }\end{array}$

Die Berechnung der Rainflow-Matrix wurde mithilfe eines Matlab-Tools realisiert. Allerdings ist der Mittel- spannungseinflussfaktor $M$ eine bauteil- und werkstoffabhängigen Größe, die für Beschleunigungsbelastungen im Gegensatz zu mechanischen Spannungen nicht bekannt ist. Dadurch ist der Mittelspannungseinfluss nicht bestimmbar. Entsprechend seiner Definition liegt er im Bereich zwischen 0 und 1 . Somit können für die jeweiligen Belastungsszenarien nur vergleichende Aussagen über das Schädigungspotential der verschiedenen Versuchsreihen getroffen werden. Insbesondere sind diese für eine vergleichende Betrachtung unterschiedlicher Fahr- und Belastungssituationen interessant [Eul06].

Der Schädigungsfaktor setzt sich aus den Mittelwerten der jeweiligen Ersatzschwingspiele zusammen, die auf die Anzahl des jeweiligen Schwingspiels normiert ist. Die berechneten Schädigungsfaktoren aus den Beschleunigungsverläufen verschiedener Gabelstapler-Nutzungsszenarien werden einander gegenübergestellt. Die Auswertung zeigt, dass vor allem in Bezug auf den Bedienhebel des Hubgerüsts für diesen Staplertyp die Überfahrt von Bodenschwellen mit mehr als $5 \mathrm{~mm}$ Höhe und einer Geschwindigkeit größer als $7 \mathrm{~km} / \mathrm{h}$ als überdurchschnittlich schädigend einzustufen ist. Die ermittelten Schädigungsfaktoren sind in Abhängigkeit von dem Mittelspannungseinflussfaktor $\mathbf{M}$ ohne Nutzmasse in Abbildung 20 zusammengestellt.

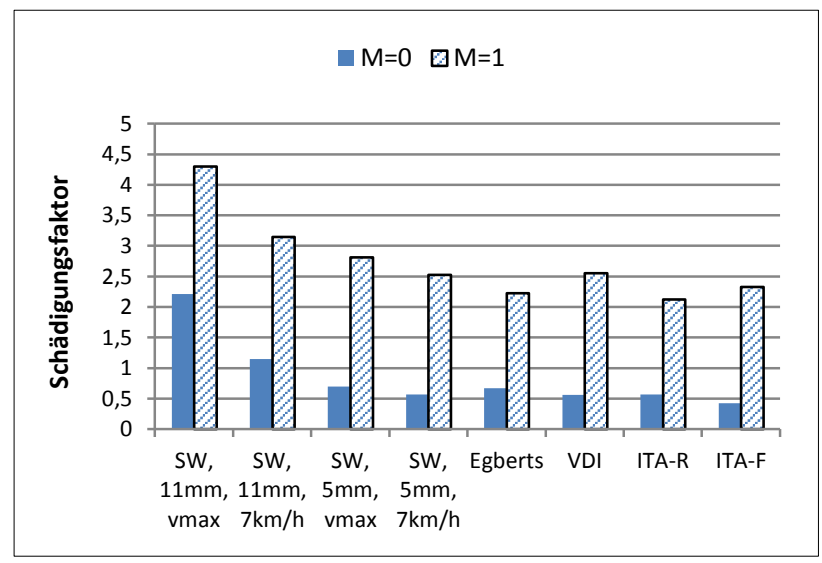

Abbildung 20. Vergleich des Schädigungsfaktors der vertikalen Beschleunigung am Bedienhebel des Hubgerüsts

Dem Diagramm ist zu entnehmen, dass die Geschwindigkeit und die Schwellenhöhe bei den Schwellenüberfahrten - wie anhand der gemessenen Beschleunigungswerte erwartet - einen erheblichen Einfluss auf das Schädigungsverhalten haben. Es ist zu erkennen, dass die Schwellenfahrten - besonders bei großer Mittelspannungsempfindlichkeit - eine deutlich größere Schädigung hervorrufen als die anderen Testszenarien. Dieses trifft auch auf die weiteren betrachteten Komponenten, wie die Antriebssteuerungen, das Fahrpedal und den Bordcomputer, zu. Das folgende Diagramm zeigt den Schädigungsfaktor des Fahrpedals bei verschiedenen Nutzmassen, Schwellenhöhen und unterschiedlichen Überfahrgeschwindigkeiten. 


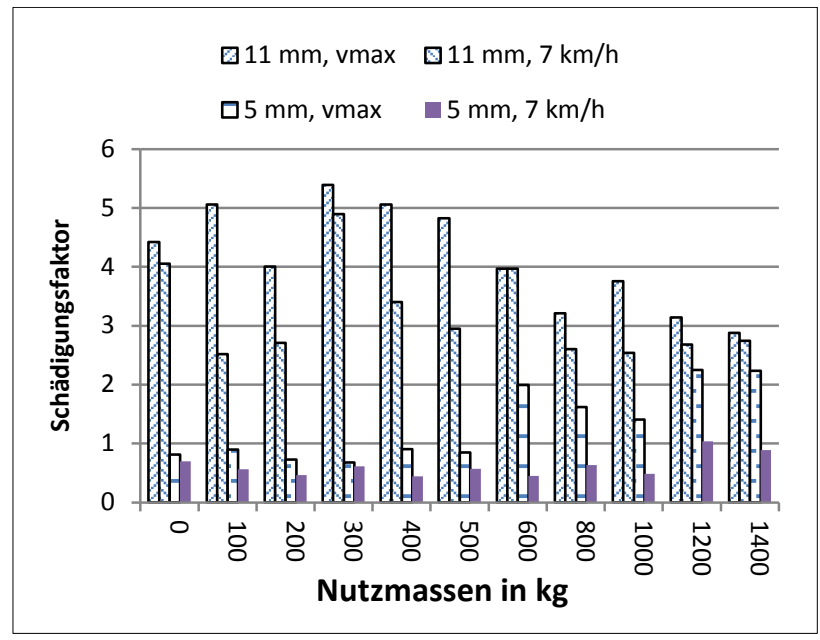

Abbildung 21. Schädigungsfaktor am Fahrpedal bei Schwellenüberfahrten für den Mittelspannungseinfluss $M=0$

Werden die Werte der Schwellenfahrt des Bedienhebels bei $\mathrm{M}=0$ aus Abbildung 20 mit der Schädigung aus Abbildung 21 des Fahrpedals ohne Nutzmasse verglichen, so ist zu erkennen, dass die Schädigungsfaktoren zum Teil um mehr als den Faktor 2 auseinander liegen. Daraus wird die Schlussfolgerung aufgestellt, dass das Fahrpedal bei einer Fahrt über eine $11 \mathrm{~mm}$ hohe Schwelle ohne Nutzmasse mehr geschädigt wird, als der Bedienhebel des Hubgerüstes. Die aus den Versuchsmessungen resultierenden Datensätze sowie die Berechnungen des Schädi- gungspotentials bestimmter Beanspruchungssituationen und Betriebsbedingungen stehen als Zwischenergebnis des Forschungsprojekts in Form von elektromechanischen Belastungskollektiven zur Verfügung.

\subsection{LEBENSDAUERUNTERSUCHUNGEN}

Um das theoretisch ermittelte Schädigungsverhalten an den Staplerkomponenten zu überprüfen und Aussagen über die zu erwartende Lebensdauer in Bezug auf die einwirkende Belastungshöhe und -häufigkeit treffen zu können, sind verschiedene Reihenuntersuchungen unter Laborbedingungen durchgeführt worden. Zur Nachstellung der mechanischen Beanspruchungen wurde ein Schwingprüfsystem errichtet, das Schwingungstests in Dauerversuchen nach den Richtlinien der DIN EN 60.068-2-6 [DIN08] ermöglicht. Abbildung 22 zeigt das an der Technischen Universität Dresden errichtete Schwingprüfsystem.

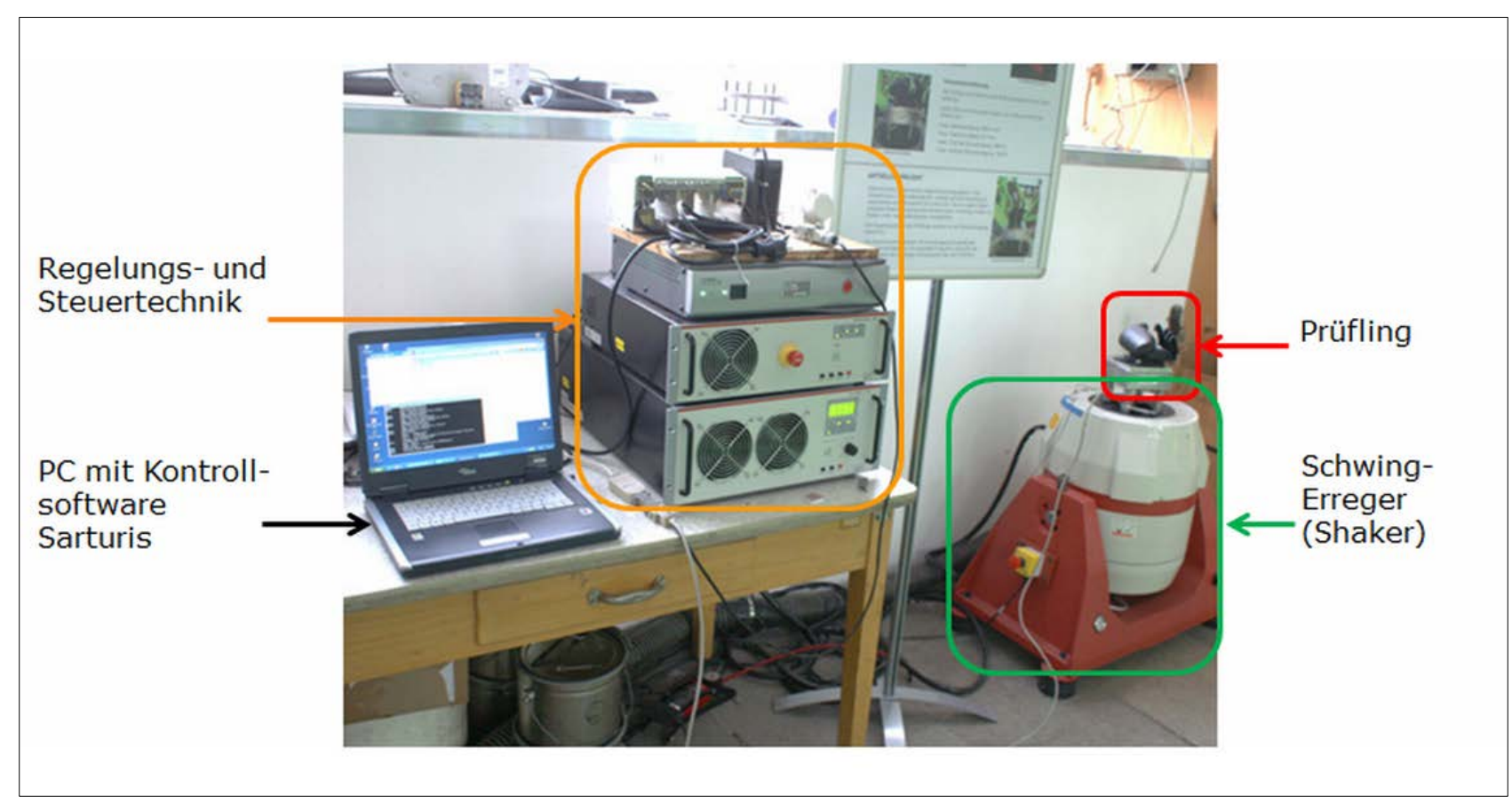

Abbildung 22. Schwingprüfsystem an der TU Dresden 
Zur Bestimmung der für die Prüfungen an den Staplerkomponenten notwendigen Parameter wurden die erfassten Beschleunigungen der Messungen am Versuchsstapler in eine Fast-Fourier-Analyse in den Frequenzbereich überführt. Des Weiteren wurde über das Schwingprüfsystem die Eigenfrequenz für jede zu testende Komponente bestimmt. Für den Bedienhebel des Hubgerüsts ergab sich aus den Frequenzverläufen der Versuchsmessungen am Schubmaststapler eine gleitende Frequenz von $40 \mathrm{~Hz}$ bis $300 \mathrm{~Hz}$. Die Eigenfrequenzermittlung für den „Solopilot“-Bedienhebel aus Abbildung 4 ergaben bis zu 3 Eigenfrequenzen, die in diesem Intervall liegen. Der vorgegebene Beschleunigungswert orientierte sich für den Bedienhebel mit $100 \mathrm{~m} / \mathrm{s}^{2}$ zunächst an den Maximalwerten der Reihenmessungen am Versuchsstapler. Anschließend ist die Beschleunigung erhöht bzw. auch bis auf $50 \mathrm{~m} / \mathrm{s}^{2}$ verringert worden. Der Funktionsstatus der Prüflinge wird während der Schwingungstests durch ein EDV-Kontrollprogramm über eine CAN-BusSchnittstelle realisiert, wodurch permanent Informationen über die Komponente an einen PC geliefert werden. Die Ergebnisse der durchgeführten Lebensdaueruntersuchungen mit dem Bedienhebel des Hubgerüstes sind in Abbildung 23 dargestellt.

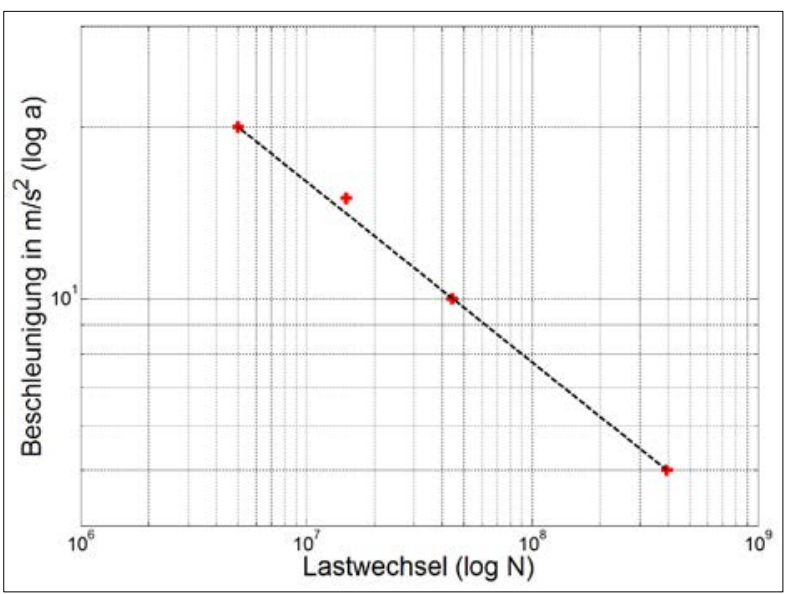

Abbildung 23. Lebensdauerdiagramm des Bedienhebels des Hubgerüsts

Das Ergebnis der Dauerschwingversuche ergibt nahezu eine Gerade, die den Zusammenhang zwischen der Belastungshöhe und den ertragbaren Lastwechseln beschreibt. Bei der gewählten Beschleunigung von $100 \mathrm{~m} / \mathrm{s}^{2}$ aus den Reihenuntersuchungen erreichte der Bedienhebel des Hubgerüstes $4,4 \cdot 10^{7}$ Lastwechsel, was innerhalb des Bereiches der Dauerfestigkeit liegt. Erst bei einer angeregten Beschleunigung von $200 \mathrm{~m} / \mathrm{s}^{2}$ liegt der Funktionsausfall mit $5 \cdot 10^{7}$ Lastwechseln im Bereich der Zeitfestigkeit. Um die Ergebnisse der Schwingungsuntersuchungen auf weitere Flurförderzeugtypen und andere mobile Arbeitsmaschinen übertragen zu können, werden die erzielten Messwerte derzeit in ein parametrisierbares Mehrkörper-Simulationsmodell überführt.

\subsection{SIMULATION DER BETRIEBSBELASTUNGEN}

Um die Auswirkungen der unterschiedlichen Belastungsgrößen auf das System Flurförderzeug - ohne konkrete Versuchsmessungen - ermitteln zu können, ist eine Modellierung des mechanischen und kinematischen Systems notwendig. Der Validierung des Modells dienen die gemessenen mechanischen Belastungen aus den Reihenuntersuchungen. Das Modell ist ein parametrisierbares Mehrkörper-Modell, das mit der Simulationssoftware „alaska“ erstellt wurde. Das Modell besteht aus über Gelenke miteinander verbundenen Körpern, dem Fahrzeugrahmen, der Fahrerkabine, dem Hubmast, des Lastaufnahmemittels und der Last.

Um beispielsweise die Auswirkungen der resultierenden Bewegung infolge einer einfachen oder einer versetzten Schwelle auf die im Projekt betrachteten Elektronikkomponenten zu ermitteln, wurden die Komponenten als Körper in das Modell integriert. Die Körper werden durch Massen und Massenträgheitsmomente beschrieben. Physikalisch gekoppelt werden die Körper durch FederDämpfer-Glieder. Der Kontakt zwischen den Rädern und der Fahrbahn wird durch Kontaktkräfte an jedem der drei Räder des Schubmaststaplers abgebildet. An den Schnittstellen Fahrerkabine und Hubmast wird der Neigezylinder über eine Feder gekoppelt. Auch die Schnittstelle von Bedienersitz und Kabine ist elastisch modelliert.

Mit diesem erstellten Modell sind alle zuvor vorgestellten Arbeitszyklen simuliert worden. Dadurch ist es möglich, weitere zeitliche Verläufe für mechanische und kinematische Größen an allen interessierenden Orten des Schubmaststaplers für das jeweilige Fahrprofil zu ermitteln. Einen Eindruck von der alaska-Simulation zeigt Abbildung 24.

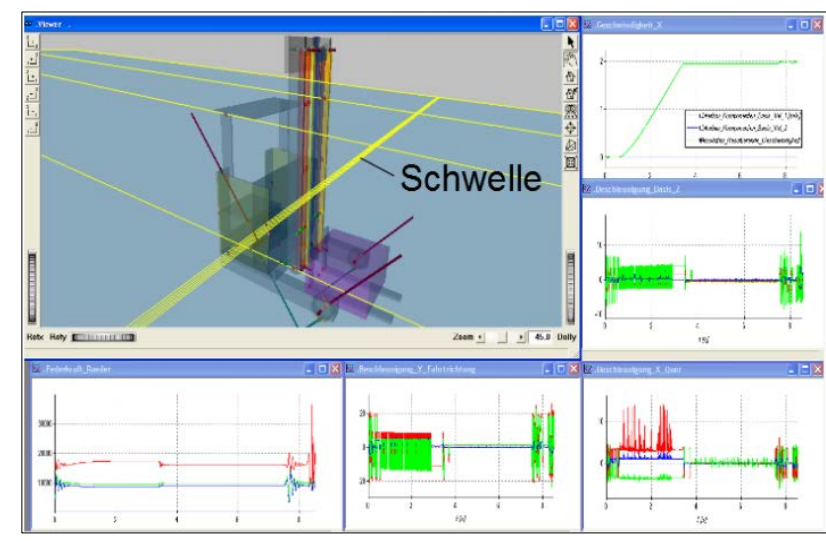

Abbildung 24. Simulationsmodell des Referenzstaplers ETV 214 am Beispiel einer Schwellenfahrt

In dieser Abbildung ist die Überfahrt des Schubmaststaplers über eine Fahrbahnschwelle zu erkennen. In diesem Beispiel sind die Beschleunigungen in allen drei Raumrichtungen am Bedienhebel des Hubmastes, die 
Fahrgeschwindigkeit sowie die Kontaktkräfte zwischen den Reifen und der Fahrbahn auf der Bedieneroberfläche dargestellt.

\section{UNTERSUCHUNG DES REGELVERHALTENS DER FAHRANTRIEBSSTEUERUNG}

An den ausgewerteten Kenngrößenverläufen der Versuchsmessungen ist deutlich $\mathrm{zu}$ erkennen, dass die Fahrantriebssteuerung aufgrund ihrer Funktion, der Leistungsversorgung und -regelung des Fahrantriebs, deutlich erkennbare Korrelationen ihrer elektrischen mit den einwirkenden mechanischen Beanspruchungen aufweist. Diese mechanischen Beanspruchungen sind dabei in erster Linie die unterschiedlichen im Betrieb auftretenden Fahrwiderstände, die entsprechend des Bedienerwunsches in Abhängigkeit von geladener Last, Fahrgeschwindigkeit und weiteren relevanten Parametern zu überwinden sind.

\subsection{THEORETISCHER LEISTUNGSBEDARF DES FAHRANTRIEBS}

Um sich selbst, den Bediener und eine gegebenenfalls aufgenommene Nutzmasse zwischen zwei Orten zu bewegen, muss das Flurförderzeug, genau wie jedes andere Landfahrzeug, mit Hilfe seiner Antriebskraft die Summe aller auftretenden Fahrwiderstände überwinden. $\mathrm{Zu}$ den aus der Fahrdynamik von Kraftfahrzeugen bekannten einzelnen Fahrwiderständen gehören der Luftwiderstand $F_{\text {Luft }}$, der Rollwiderstand $F_{\text {Roll, }}$, der Steigungswiderstand $F_{\text {Steigung }}$ und der Beschleunigungswiderstand $F_{\text {Beschleunigung }}$ [BS01].

Bewegt sich ein Körper relativ in einem flüssigen oder gasförmigen Medium, erfährt er einen Strömungswiderstand, der entgegen der Relativbewegung wirkt. Ein sich durch die Umgebungsluft bewegendes Fahrzeug ist entsprechend dem Luftwiderstand $F_{\text {Luft }}$ ausgesetzt.

$$
\mathrm{F}_{\text {Luft }}=\frac{1}{2} \cdot \mathrm{c}_{\mathrm{w}} \cdot \mathrm{A} \cdot \rho_{\text {Luft }} \cdot \mathrm{v}_{\text {relativ }}^{2}
$$

Er steigt mit der Relativgeschwindigkeit $v_{\text {relativ }}$ vom Fahrzeug $v_{\text {Fahrzeug }}$ zur Umgebungsluft $v_{\text {Luft }}$ quadratisch an und ist zudem von der Beschaffenheit des Fahrzeuges, charakterisiert durch seinen Strömungskoeffizienten $c_{w}$ und der Stirnfläche $A$, abhängig. Die Stirnfläche $A$, die sich aus der Projektion des Fahrzeugs auf eine Ebene senkrecht zur Fahrbahn ergibt, ist in Abbildung 25 dargestellt.

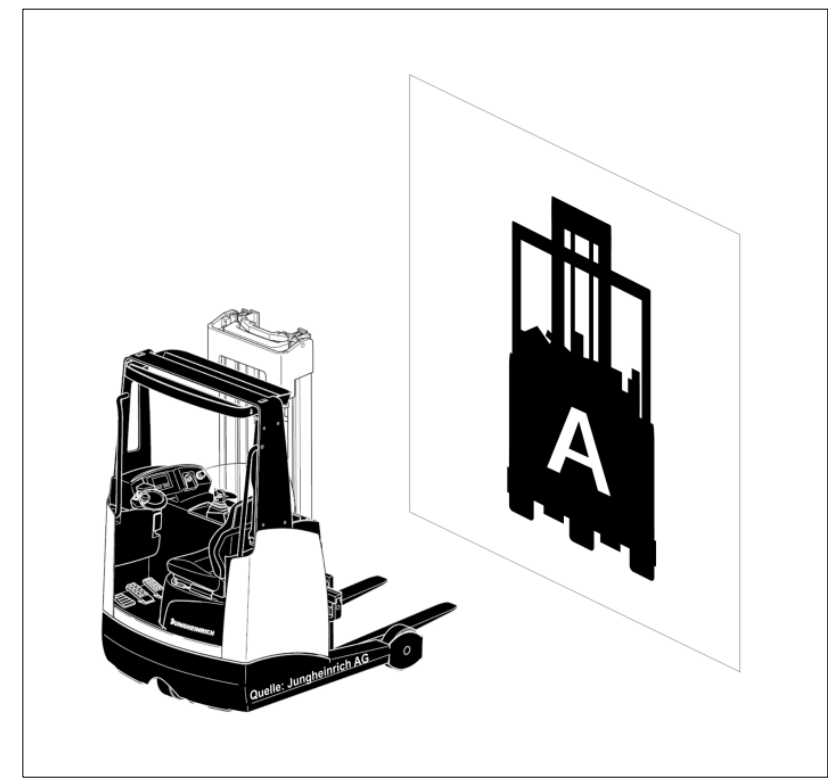

Abbildung 25. Stirnfläche A des Schubmaststaplers

Obwohl Flurförderzeuge durch ihre Bauhöhe eine große Stirnfläche $A$ sowie einen, im Vergleich mit anderen Landfahrzeugen, eher hohen Strömungskoeffizienten $c_{w}$ aufweisen, spielt der Luftwiderstand aufgrund der geringen maximalen Fahrgeschwindigkeit für Flurförderzeuge nur eine untergeordnete Rolle.

Der Rollwiderstand $F_{\text {Roll }}$ ist abhängig von der Masse des Fahrzeuges $m_{F z}$, seiner Zuladung $m_{Z u l}$ und dem Rollwiderstandskoeffizient $f_{\text {Roll }}$.

$$
\mathrm{F}_{\mathrm{Roll}}=\left(\mathrm{m}_{\mathrm{Fz}}+\mathrm{m}_{\mathrm{Zul}}\right) \cdot \mathrm{g} \cdot \mathrm{f}_{\mathrm{Roll}} \cdot \cos (\alpha)
$$

Abbildung 26 zeigt die wirkenden Kräfte aufgrund des Rollwiderstandes, der aufgrund der elastischen Verformungen von Reifen und Fahrbahn in deren Kontaktstellen auftritt.

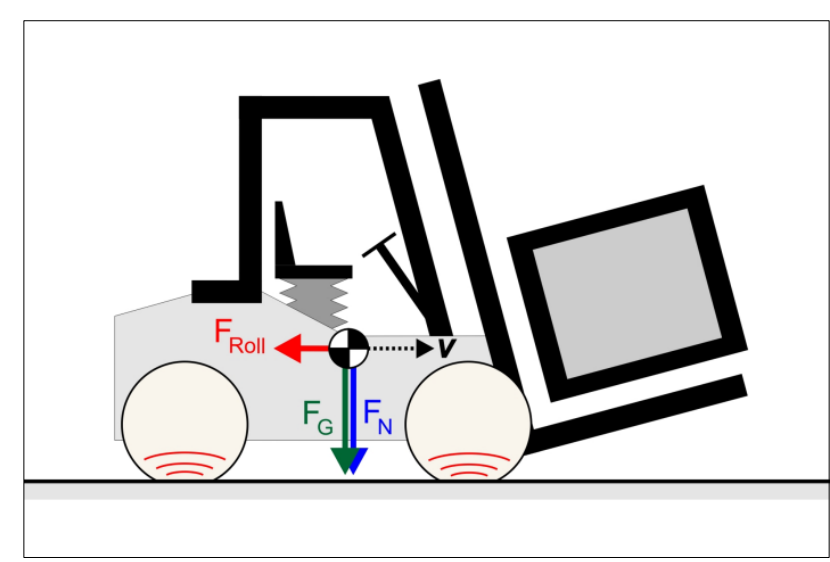

Abbildung 26. Betrachtung des Rollwiderstandes

Wird eine Steigung mit einem Rampenwinkel $\alpha$ befahren, verändert sich der Rollwiderstand um den Faktor 
$\cos (\alpha)$. Zusätzlich tritt der Steigungswiderstand $F_{\text {Steigung }}$ auf.

$$
\mathrm{F}_{\text {Steigung }}=\left(\mathrm{m}_{\mathrm{Fz}}+\mathrm{m}_{\mathrm{Zul}}\right) \cdot \mathrm{g} \cdot \sin (\alpha)
$$

Die in einer Steigung auftretenden Kräfte sind in Abbildung 27 dargestellt.

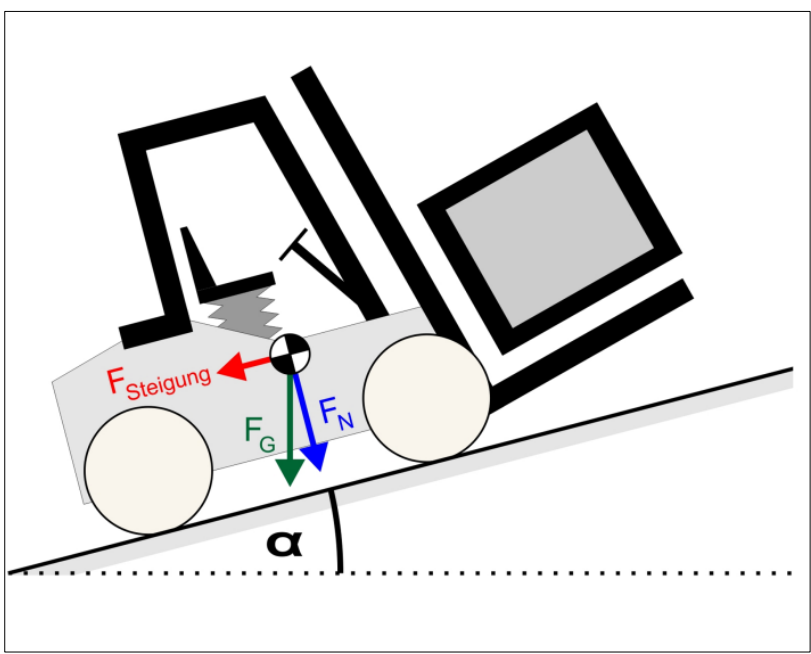

Abbildung 27. Steigungswiderstand in Abhängigkeit des Rampenwinkels $\alpha$

Soll das Fahrzeug beschleunigt werden, müssen zudem die Trägheitsmomente der durch den Antrieb zu beschleunigenden rotierenden Bauteile, wie beispielweise Räder oder Antriebswellen, überwunden werden. Diese Trägheitsmomente werden bei dem Beschleunigungswiderstand $F_{\text {Beschleunigung }}$ durch den dimensionslosen Massenfaktor $e_{i}$ berücksichtigt.

$$
\mathrm{F}_{\text {Beschleunigung }}=\left(\mathrm{e}_{\mathrm{i}} \cdot \mathrm{m}_{\mathrm{Fz}}+\mathrm{m}_{\mathrm{Zul}}\right) \cdot \mathrm{a}
$$

Abbildung 28 stellt den Beschleunigungswiderstand am Flurförderzeug dar.

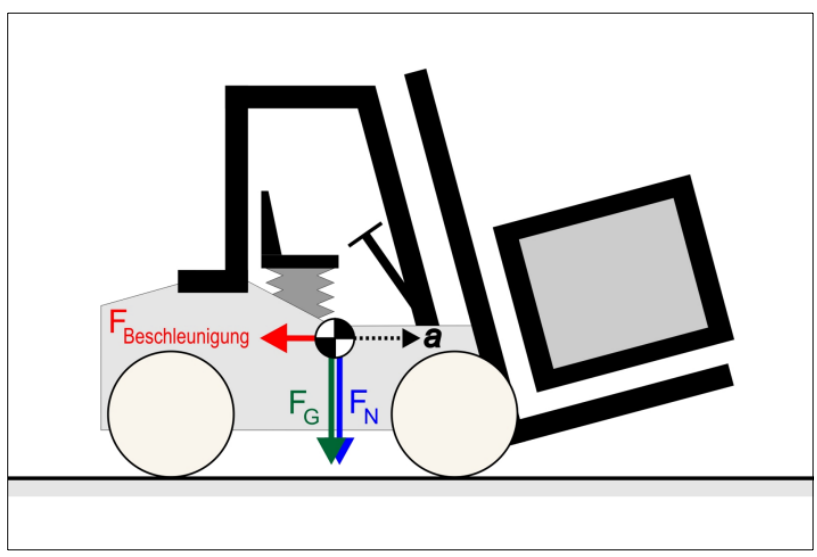

Abbildung 28. Wirkende Kräfte bei der Beschleunigung

Da die Überfahrt von Fahrbahnschwellen mit diesen, aus der Literatur bekannten, Fahrwiderstandskomponenten nicht abzubilden ist, wird sie als besondere Beanspru- chungssituation gesondert anhand eines mechanischen Ersatzmodelles betrachtet. Idealisiert wurde dabei von starren Körpern und einer starren Kopplung der einzelnen Elemente zueinander ausgegangen. Abbildung 29 zeigt das Auftreffen eines Rades auf die Fahrbahnschwelle.

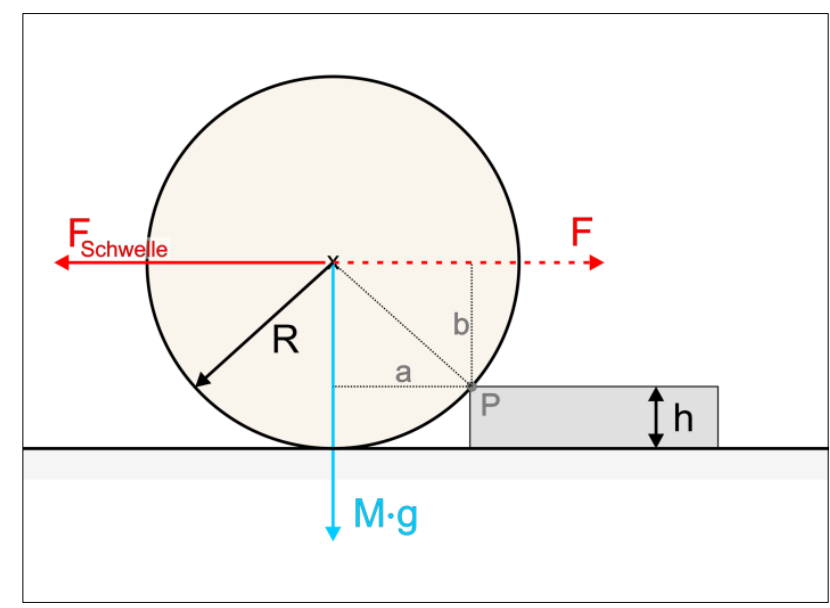

Abbildung 29. Notwendige Kraft F zur Überwindung einer Fahrbahnschwelle

Soll das Rad, beziehungsweise die Fahrzeugachse, nun über die Fahrbahnschwelle mit der Höhe $h$ bewegt werden, ergibt sich für den Zeitpunkt des Auftreffens auf die Fahrbahnschwelle eine zusätzlich zu den anderen Fahrwiderständen wirkende benötigte Widerstands-

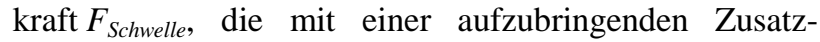
kraft $F^{`}$ auszugleichen ist.

$$
\mathrm{F}_{\text {Schwelle }}=\frac{\mathrm{M} \cdot \mathrm{g} \cdot \mathrm{a}}{\mathrm{b}}
$$

Durch Substitution der Strecken $a$ und $b$ durch den Radius des überfahrenden Rades bzw. der überfahrenden Räder $R$ und der Schwellenhöhe $h$ entsprechend der Formeln

$$
\begin{aligned}
& \mathrm{a}=\sqrt{\mathrm{R}^{2}-\mathrm{b}^{2}} \text { und } \\
& \mathrm{b}=\mathrm{R}-\mathrm{h}
\end{aligned}
$$

ergibt sich für den Schwellenwiderstand $F_{\text {Schwelle: }}$

$$
\mathrm{F}_{\text {Schwelle }}=\frac{\mathrm{M} \cdot \mathrm{g} \cdot \sqrt{\mathrm{R}^{2}-(\mathrm{R}-\mathrm{h})^{2}}}{(\mathrm{R}-\mathrm{h})} .
$$

Für eine exakte Berechnung der Schwellenwiderstandskraft $F_{\text {Schwelle }}$ ist zu berücksichtigen, dass sich die Rad- bzw. Achslast $M$ durch das Aufsteigen auf die Fahrbahnschwelle, und die dadurch resultierende leichte Fahrzeugneigung, je nach Schwerpunktlage des Gesamtfahrzeugs geringfügig verringert. Abbildung 30 zeigt die wirkenden Kräfte inmitten der Schwellenauffahrt. 


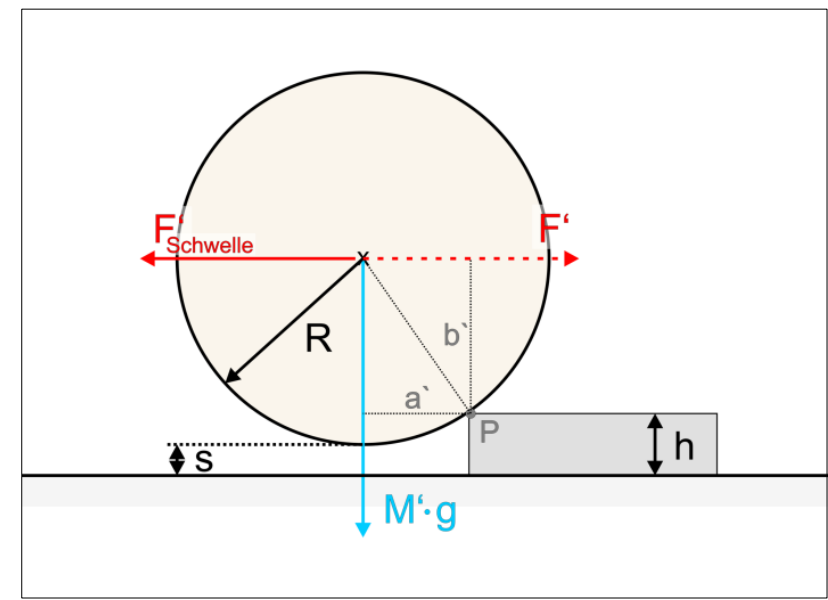

Abbildung 30. Während der Überfahrt sich verändernde Kraft $F^{`}$ zur Überwindung der Fahrbahnschwelle

Anhand der sich nun veränderten geometrischen Verhältnisse

$$
\begin{aligned}
& \mathrm{a}^{\prime}<\mathrm{a} \text { und } \\
& \mathrm{b}^{\prime}>\mathrm{b}
\end{aligned}
$$

ergibt sich für die in dem abgebildeten Zeitpunkt aufzubringende Kraft $F^{`}$ entsprechend

$$
\mathrm{F}^{\prime}<\mathrm{F} \text {. }
$$

Steht das Rad bzw. die Achse, wie in Abbildung 31 dargestellt, senkrecht über der Schwellenkante, ist die Schwellenauffahrt beendet und der Schwellenwiderstand errechnet sich entsprechend zu Null.

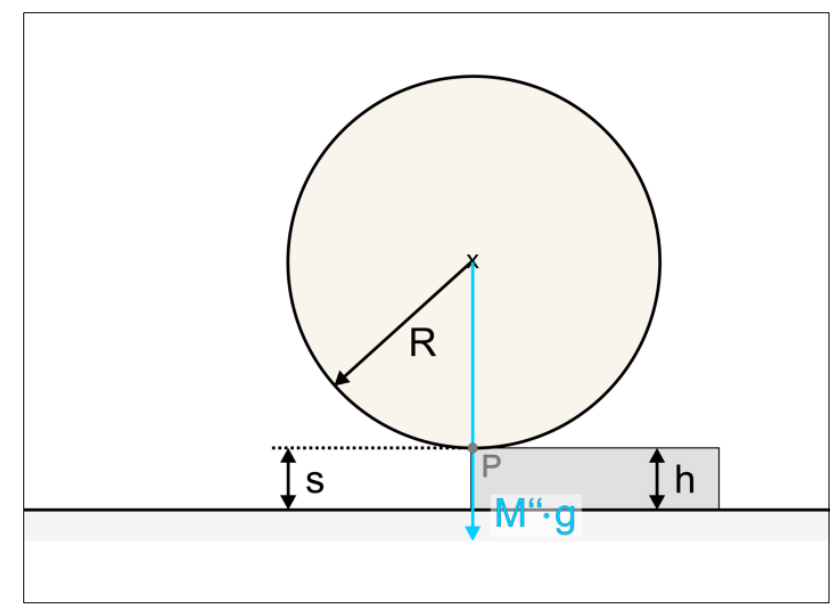

Abbildung 31. Überwundene Fahrbahnschwelle

Der gesamte Verlauf der Kraft $F^{(c)}$ ist ohne die Berücksichtigung der sich minimal verändernder Rad- bzw. Achslast $M^{(c)}$ für das Aufsteigen eines Flurförderzeugs mit einer Achslast von $1.180 \mathrm{~kg}$ auf eine $10 \mathrm{~mm}$ hohe Fahrbahnschwelle berechnet worden (siehe Abbildung 32).

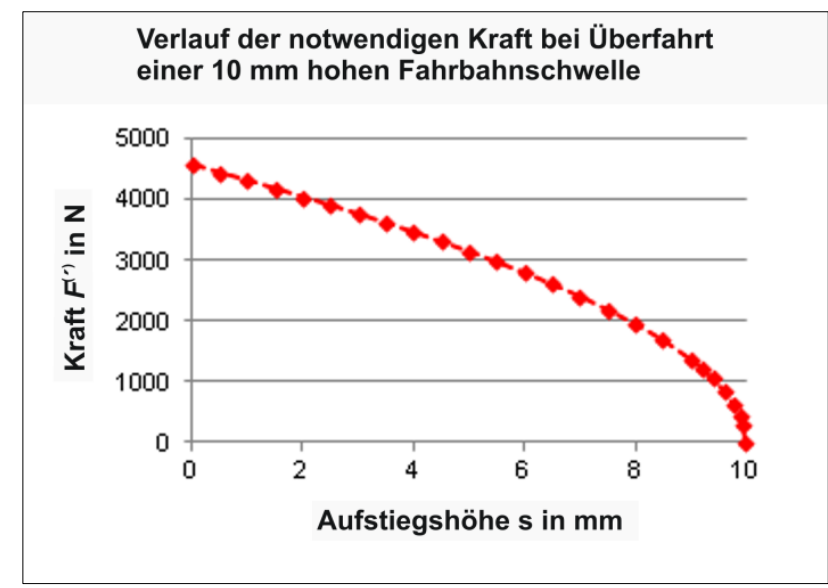

Abbildung 32. Verlauf der zur Schwellenüberfahrt benötigten Kraft $F(')$

Auch an diesem Diagramm ist zu erkennen, dass die zur Schwellenüberfahrt zusätzlich benötigte Kraft während des Auftreffens des Rades bzw. der Achse am höchsten ist und im Verlauf des Aufsteigens stetig abnimmt bis das Hindernis überwunden ist.

Entsprechend der einzelnen aufgezeigten Fahrwiderstandskomponenten ergibt sich durch ein Aufsummieren die gesamte Fahrwiderstandskraft $F_{\text {Fahrwiderstand: }}$

$\mathrm{F}_{\text {Fahrwiderstand }}=\mathrm{F}_{\text {Luft }}+\mathrm{F}_{\text {Roll }}+\mathrm{F}_{\text {Steigung }}+\mathrm{F}_{\text {Beschleunigung }}+\mathrm{F}_{\text {Schwelle }}$.

Um unter Einwirkung dieser Fahrwiderstandskraft eine bestimmte Geschwindigkeit $v$ zu erreichen, ist folgende Antriebsleistung $P_{\text {Antrieb,erf. }}$ notwendig:

$$
\begin{aligned}
& \mathrm{P}_{\text {Antrieb, erf. }}= \\
& \left(\mathrm{F}_{\text {Luft }}+\mathrm{F}_{\text {Roll }}+\mathrm{F}_{\text {Steigung }}+\mathrm{F}_{\text {Beschleunigung }}+\mathrm{F}_{\text {Schwelle }}\right) \cdot \mathrm{v} .
\end{aligned}
$$

\subsection{VERGLEICH DER ERMITTELTEN BELASTUNGEN}

Bei der Auswertung der in den umfangreichen Versuchsmessungen mit dem Referenzstapler an der Fahrantriebssteuerung aufgezeichneten elektrischen Kenngrößenverläufen konnten die einzelnen Fahrwiderstände, wie der Beschleunigungswiderstand und auch (zusammengefasst) Roll- und Luftwiderstand, identifiziert werden (vgl. Abbildung 33). 


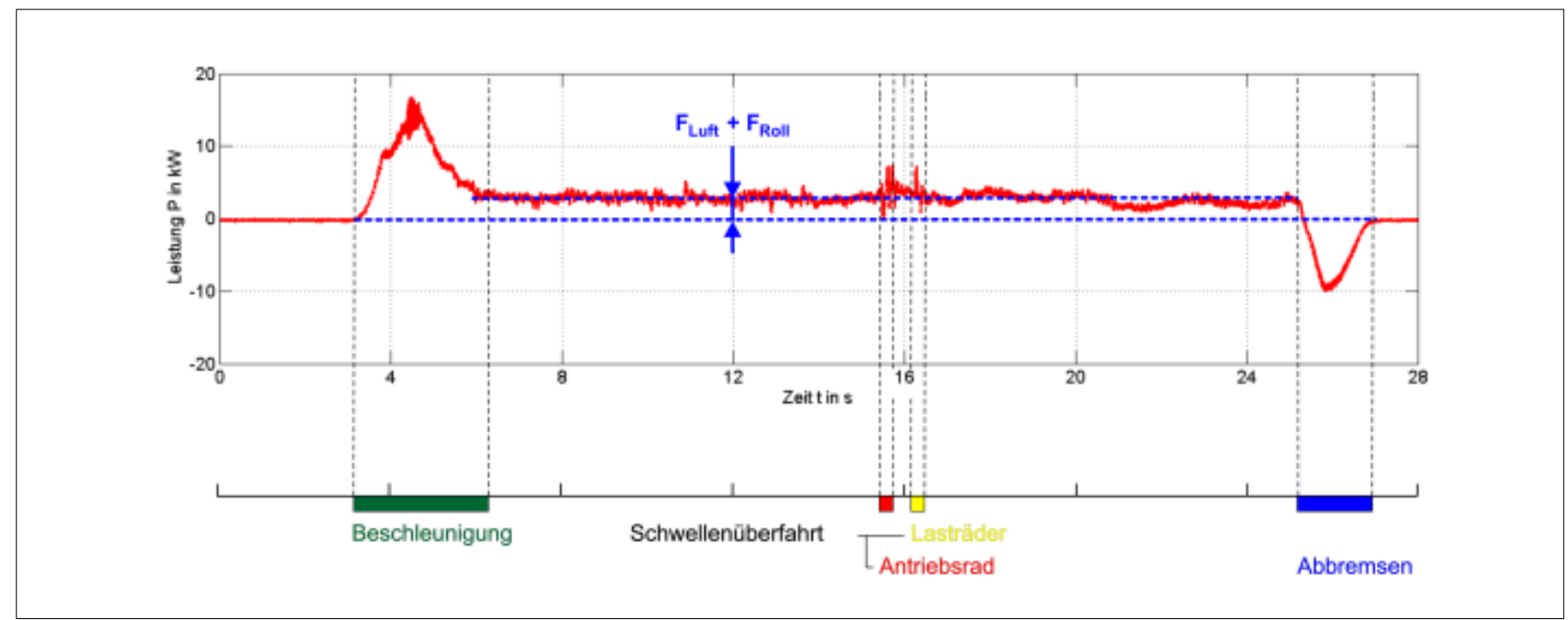

Abbildung 33. Analyse des Roll- und Luftwiderstandes

Bei dem Vergleich des Schwellenwiderstandes fällt unterdessen auf, das sich die von der Fahrantriebssteuerung geregelte Antriebsleistung beim Auftreffen auf das Hindernis sehr schnell stark erhöht, was zu einem deutlichen Überschwinger und einem darauf folgenden Einschwingvorgang führt. Abbildung 34 zeigt den Verlauf der von der Fahrantriebssteuerung umgesetzten Leistung im Vergleich zu der aus der Fahrwiderstandsbetrachtung (Abschnitt 4.1) theoretisch ermittelten benötigten Antriebsleistung während der Schwellenüberfahrt.

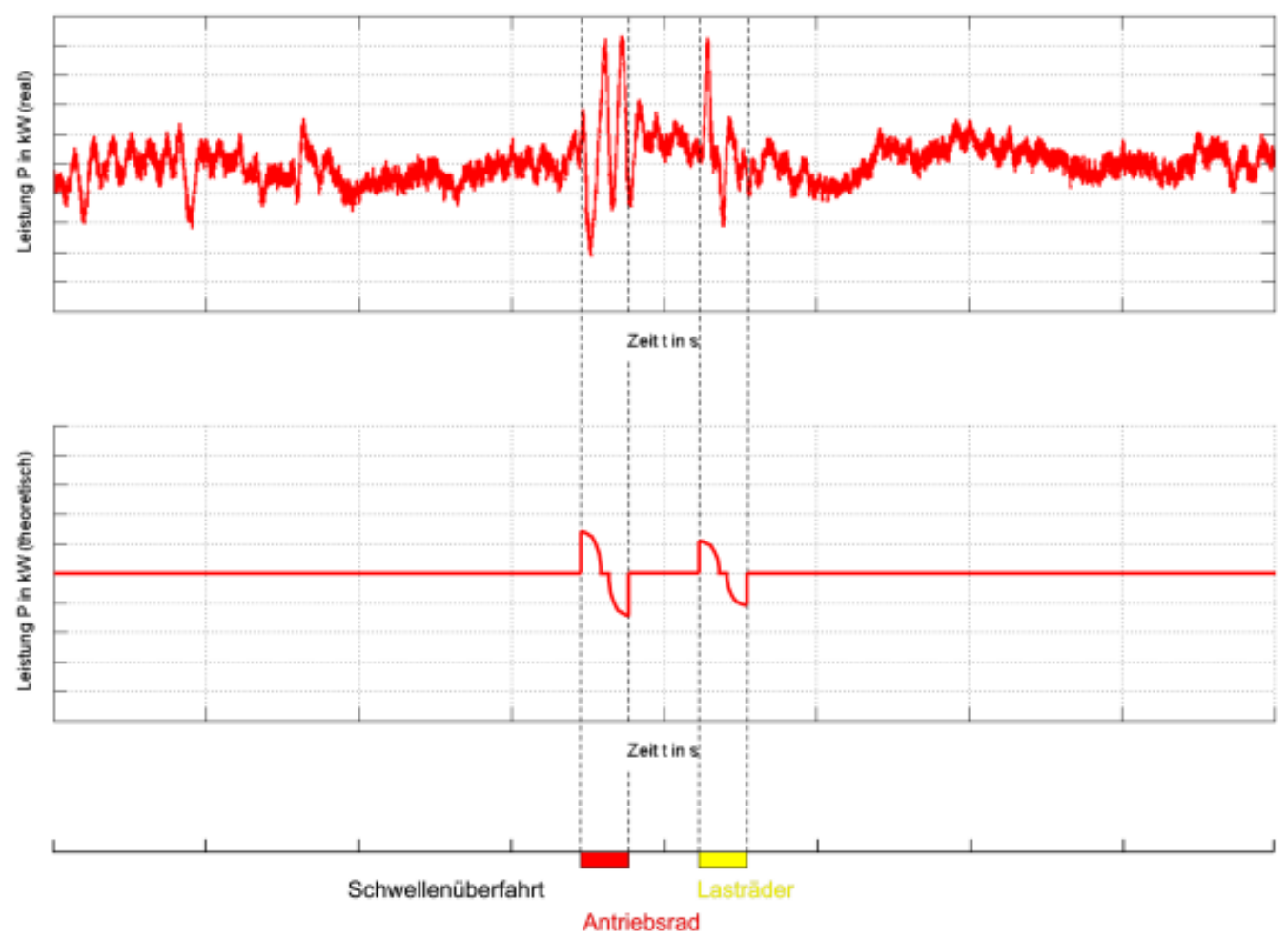

Abbildung 34. Gemessene Leistungsregelung und tatsächlicher Leistungsbedarf bei einer Schwellenüberfahrt 
Um genauere Vorhersagen über die am Flurförderzeug auftretenden elektrischen Beanspruchungen aufgrund von Fahrbahnschwellen und anderen auftretenden Hindernissen treffen zu können, sind nähere Kenntnisse über das Regelverhalten der Fahrantriebssteuerung notwendig. Aktuell wird daher an der Aufstellung eines Regelkreises und dem Übertragungsverhalten von Stell- und Störgrößen gearbeitet. Abbildung 35 zeigt schematisch einen solchen Regelkreis.

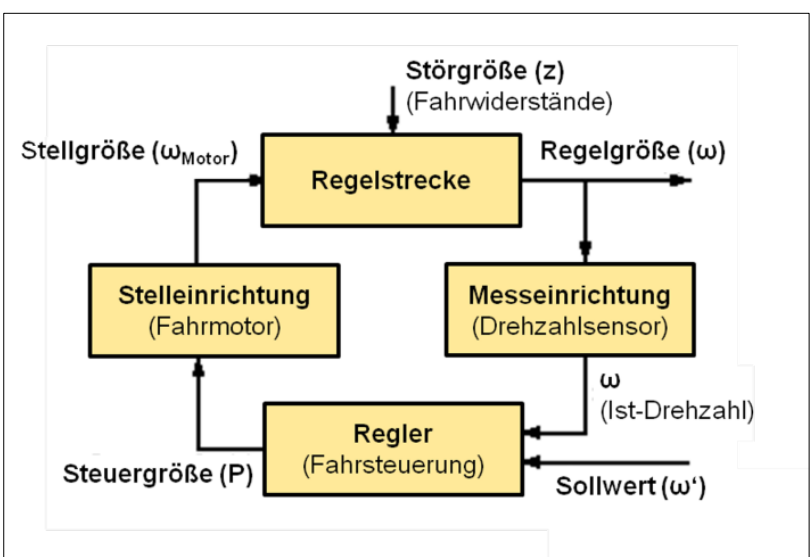

Abbildung 35. Schema eines Regelkreises für den Fahrantrieb

\subsection{UNTERSUCHUNGEN AM ANTRIEBSSTRANG}

Um das Regelverhalten der Fahrantriebssteuerung unter zuvor definierten Beanspruchungsbedingungen näher charakterisieren zu können, wurde zudem ein Flurförderzeug-Antriebsprüfstand konzipiert und aufgebaut.
Der Prüfstand enthält den gesamten Antriebsstrang des Referenzstaplers. Die Energieversorgung des Antriebs übernimmt entsprechend eine Traktionsbatterie mit $48 \mathrm{~V}$ Nennspannung. Hieran angeschlossen ist die Fahrantriebssteuerung, die entsprechend ihres Regelzustandes die Energie umwandelt und an den 3-Phasen-Wechselstromantrieb weiterleitet. Die für die Regelung benötigten Signale werden im Gegensatz zum realen Versuchsfahrzeug dabei nicht über Bedieneinrichtungen, wie Pedale oder ähnlichem, sondern über ein angeschlossenes Datenverarbeitungssystem vorgegeben. Eine gekoppelte Lastmaschine ermöglicht die Einspielung beliebiger Lastmomentverläufe, die über einen analogen Eingangskanal am Laststrang vorgegeben werden. Abbildung 36 zeigt die wesentlichen Bestandteile des Flurförderzeug-Antriebsprüfstandes.

Neben der Untersuchung des Regelverhaltens bietet der Prüfstand damit die Möglichkeit, Lebensdauertests unter vorgegebenen Beanspruchungssituationen, gekennzeichnet durch entsprechende Drehzahl- und Drehmomentverläufe, durchführen zu können.

Durch eine entsprechende Vorgabe von Kenngrößenverläufen werden derzeit Untersuchungen mit theoretisch endlos aneinandergereihten Schwellenüberfahrten durchgeführt. Hierbei wird untersucht, ob das durch die aufgezeigten Überschwinger gekennzeichnete Regelverhalten der Fahrantriebssteuerung schädigende und lebensdauerverkürzende Auswirkungen auf die Antriebskomponenten des Flurförderzeuges hat.

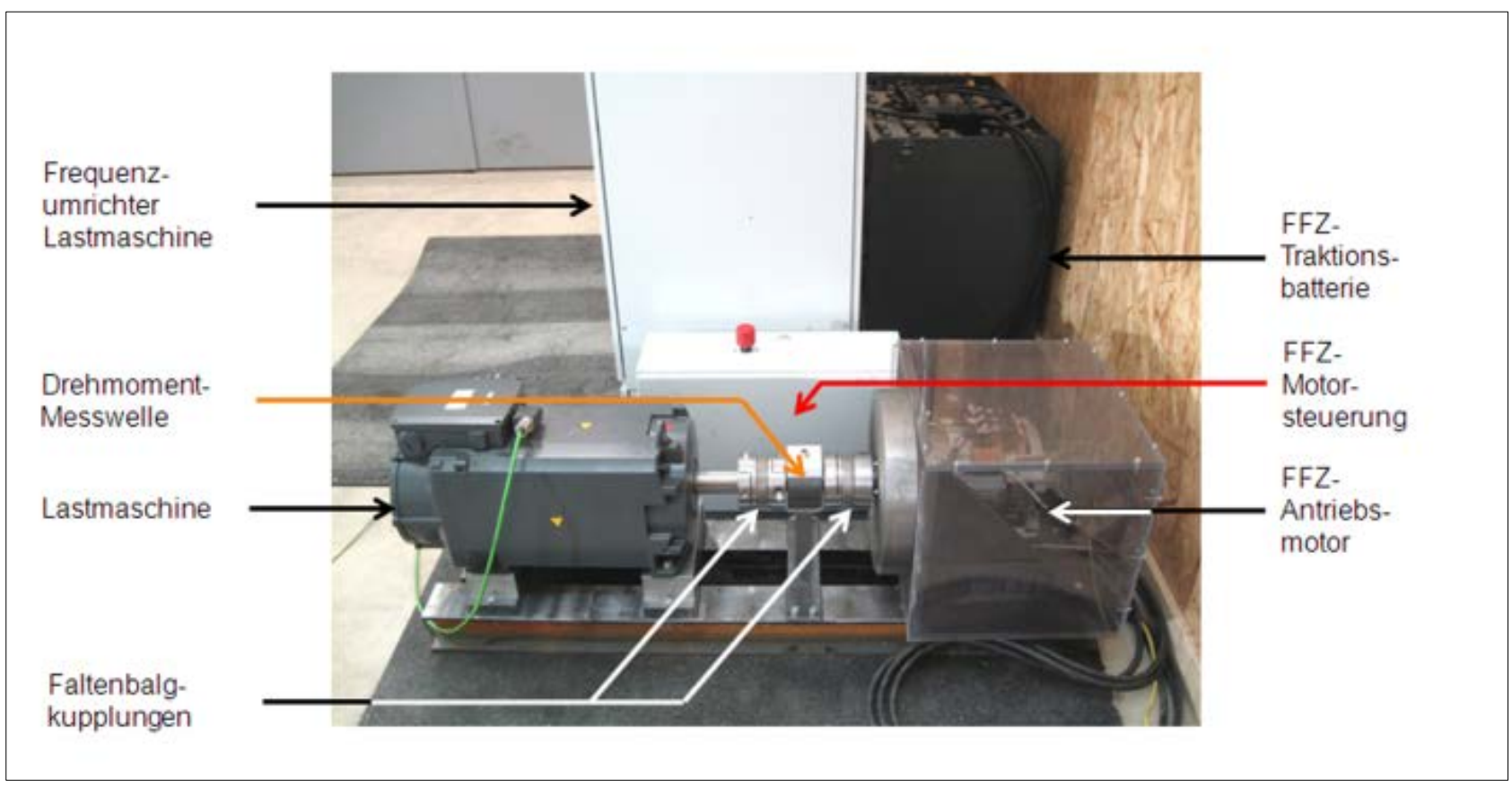

Abbildung 36. Übersicht des Flurförderzeug-Antriebsprüfstandes 


\section{5}

\section{ZUSAMMENFASSUNG UND AUSBLICK}

Im Rahmen des Forschungsvorhabens „Dimensionierung elektronischer Komponenten in Flurförderzeugen" wurde anhand einer Schwachstellenanalyse ein Elektro-Schubmaststapler für die Untersuchung von einwirkenden Belastungen und deren Folgen auf die verbauten Elektronikkomponenten ausgewählt. In Reihenuntersuchungen mit verschiedenen bekannten und neu aufgestellten Fahrprofilen wurden an einem von der Jungheinrich AG zur Verfügung gestellten Versuchsstapler elektrische und mechanische Kenngrößenverläufe messtechnisch aufgezeichnet und ausgewertet. Hinsichtlich der mechanischen Schwing- und Stoßbelastungen auf die einzelnen Komponenten wurde eine Bewertung hinsichtlich ihres Schädigungsvermögens mit der aus der Betriebsfestigkeit bekannten Rainflow-Klassierung durchgeführt.

Die Nachstellung und gezielte Überhöhung der gemessenen mechanischen Beanspruchungen erfolgte an einem aufgebauten elektro-dynamischen Schwingprüfsystem, an dem Lebensdauertests des Hubmast-Bedienhebels für unterschiedliche Beschleunigungen durchgeführt wurden. Hieraus wurde eine Ausfallcharakteristik in Abhängigkeit von Beanspruchungshöhe und -häufigkeit aufgestellt. Weitere Komponenten des Referenzstaplers werden derzeit mit Hilfe des Systems untersucht.

Bei der Betrachtung der gemessenen elektrischen Kenngrößenverläufe fiel insbesondere das Regelverhalten der Fahrantriebssteuerung auf, die den Drehstromfahrantrieb nach Bedienbefehl und den auftretenden Fahrwiderständen mit einer bestimmten elektrischen Leistung ansteuert. Hierbei ist insbesondere bei der Schwellen- bzw. Hindernisüberfahrt eine im Vergleich zu den berechneten theoretischen Kenngrößenverläufen deutlich abweichende und durch große Amplituden im Schwingungsverlauf gekennzeichnete Regelung aufgefallen. Um Rückschlüsse auf eine mögliche Schädigung der Antriebskomponenten durch dieses Verhalten ziehen zu können, werden derzeit Lebensdaueruntersuchungen an einem FlurförderzeugAntriebsprüfstand durchgeführt, bei denen durch eine entsprechende Drehzahl- und Lastmomentvorgabe endlose Schwellenüberfahrten simuliert werden.

Des Weiteren werden die in den Versuchsmessungen aufgenommenen elektrischen und mechanischen Kenngrößenverläufe für die Fahrantriebssteuerung auf Korrelation zwischen diesen beiden Beanspruchungsarten im Zeit- und Frequenzbereich hin untersucht. Abschließende Untersuchungen von betriebenen Komponenten auf dem Schwingprüfsystem sollen die Lebensdauer einzelner Komponenten unter der zeitgleichen Einwirkung elektrischer und mechanischer Beanspruchungen im Vergleich zu einer getrennten Betrachtung aufzeigen.
[BS01] Braess, Hans-Hermann; Seiffert, Ulrich: Vieweg Handbuch Kraftfahrzeugtechnik. 2. Auflage. Braunschweig, Wiesbaden: Friedrich Vieweg \& Sohn Verlagsgesellschaft mbH, 2001

[DIN08] DIN Deutsches Institut für Normung e.V. (Hrsg.): DIN EN 60.068-2-6: Umgebungseinflüsse Teil 2-6: Prüfverfahren - Prüfung Fc: Schwingungen (sinusförmig). Berlin: Beuth-Verlag, 2008

[DIN09] DIN Deutsches Institut für Normung e.V. (Hrsg.): DIN EN 13.059:2009-06: Sicherheit von Flurförderzeugen Schwingungsmessung; Deutsche Fassung EN 13059:2002+A1:2008. Berlin: Beuth-Verlag, 2009

[Egb00] Egberts, Theo: Gabelstaplertest „Transport + Opslag“ und „Fördern und Heben“. In: Tagungsband zur 3. Hamburger Staplertagung. Hamburg: Helmut-Schmidt-Universität, 2000

[Eul06] Eulitz, Klaus-Georg.: Betriebsfestigkeit. Studienbrief. TU Dresden, Fakultät Maschinenwesen, Arbeitsgruppe Fernstudium, 2006

[SWSO10] Schmidt, Stephanie; Weigelt, Stefan; Schmidt, Thorsten; Overmeyer, Ludger: Ermittlung und Analyse elektromechanischer Belastungskollektive an elektronischen Komponenten in Flurförderzeugen. In: Tagungsband zum 6. Fachkolloquium der WGTL. Garbsen: PZH Produktionstechnisches Zentrum GmbH, 2010, S. 221-232

[VDI02] VDI-Gesellschaft Produktion und Logistik (Hrsg.): VDI 2.198: Typenblätter für Flurförderzeuge. Berlin: BeuthVerlag, 2002

[VDI10] VDI-Gesellschaft Produktion und Logistik (Hrsg.): VDI 4.461: Beanspruchungskategorien für Gabelstapler. Berlin: Beuth Verlag, 2010

[WO11] Weigelt, Stefan; Overmeyer, Ludger: Elektronikkomponenten von Gabelstapler auf dem Prüfstand. Belastungen im täglichen Betrieb. In: Hebezeuge Fördermittel, Berlin 51, 2011, 4, S. 188-191 


\section{FÖRDERHINWEIS}

Das IGF-Vorhaben 16228 BG/1 der Forschungsvereinigung Intralogistik/ Fördertechnik und Logistiksysteme e.V. (FG IFL) wurde über die AiF im Rahmen des Programms zur Förderung der industriellen Gemeinschaftsforschung (IGF) vom Bundesministerium für Wirtschaft und Technologie aufgrund eines Beschlusses des Deutschen Bundestages gefördert.
Gefördert durch:

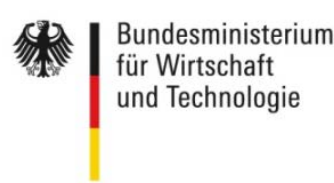

aufgrund eines Beschlusses des Deutschen Bundestages 